\title{
Phytochromes B1/B2 Are Major Regulators of Ripening-associated Epigenome Reprogramming in Tomato Fruits
}

\section{Ricardo Bianchetti}

Departamento de Botânica, Instituto de Biociências, Universidade de São Paulo, São Paulo, Brasil. https://orcid.org/0000-0001-8739-5460

\section{Nicolas Bellora}

Instituto Andino Patagónico de Tecnologias Biológicas y Geoambientales - CCT-CONICET, Patagonia Norte, Bariloche, Argentina. https://orcid.org/0000-0001-6637-3465

\section{Luis de Haro}

Weizmann Institute of Science https://orcid.org/0000-0003-0668-248X

\section{Rafael Zuccarelli}

Departamento de Botânica, Instituto de Biociências, Universidade de São Paulo, São Paulo, Brasil.

\section{Daniele Rosado}

Cold Spring Harbor Laboratory https://orcid.org/0000-0002-9319-9041

\section{Luciano Freschi}

Departamento de Botânica, Instituto de Biociências, Universidade de São Paulo, São Paulo, Brasil.

\section{Magdalena Rossi}

Universidade de São Paulo https://orcid.org/0000-0003-3650-772X

Luisa Bermudez ( $\sim$ bermudez.luisa@inta.gob.ar)

IABIMO-CONICET https://orcid.org/0000-0002-9905-6287

\section{Article}

Keywords: carotenoid, chlorophyll, DNA methylation, epigenetics, fleshy fruit, phytochrome, RNA-seq, RdDM, tomato

Posted Date: August 5th, 2020

DOI: https://doi.org/10.21203/rs.3.rs-50917/v1

License: (c) (1) This work is licensed under a Creative Commons Attribution 4.0 International License. Read Full License 


\section{Abstract}

Phytochrome-mediated light and temperature perception has been shown to be a major regulator of fruit development. Furthermore, chromatin remodelling via DNA demethylation has been described as a crucial mechanism behind the fruit ripening process; however, the molecular basis underlying the triggering of this epigenetic modification remains largely unknown. Here, through integrative analyses of the methylome, siRNAome and transcriptome of tomato fruits from phyA and phyB1B2 null mutants, we report that PHYB1 and PHYB2 control genome-wide DNA methylation during fruit development. The experimental evidence indicates that PHYB1B2 signal transduction is mediated by the coordinated expression of DNA methylases/demethylases, histone-modifying enzymes and chromatin remodelling factors, resulting in the transcriptional regulation of photosynthetic and ripening-associated genes. This new level of understanding provides insights into the orchestration of epigenetic mechanisms in response to environmental cues affecting agronomical traits.

\section{Introduction}

As sessile organisms, plants must constantly monitor their environment and continuously tune their gene expression to enable adaptation and survival ${ }^{1}$. Light is one of the main environmental cues that controls plant growth and development from seed germination to senescence ${ }^{2}$. Plants employ different photoreceptors to detect and respond to changes in the incident spectral composition (from UV-B to farred wavelengths), light direction and photoperiod. These photoreceptor families include (i) phytochromes (PHYs), which perceive red/far-red (R/FR) light; (ii) cryptochromes (CRYs), phototropins, and 'Zeitlupes', which sense blue/UV-A light; and (iii) the UV-B receptor UVR8 ${ }^{3}$.

After photoreceptor activation, complex signal transduction pathways control the expression of lightregulated genes via transcriptional, posttranscriptional, and posttranslational mechanisms ${ }^{2}$. Several hub proteins in the light signal transduction pathway triggered by PHYs, CRYs and UVR8 have been identified, including transcription factors (TFs) such as PHY-INTERACTING FACTORS (PIFs) and ELONGATED HYPOCOTYL5 (HY5) as well as the ubiquitin E3 ligase complex comprising CONSTITUTIVE PHOTOMORPHOGENIC1 (COP1) ${ }^{2}$. Additionally, the effect of light on alternative splicing (AS) has recently been described ${ }^{4}$. In Arabidopsis thaliana, 1,505 genes undergo changes in the AS levels of their transcripts within $1 \mathrm{~h}$ of exposure to red light in a PHY-dependent manner ${ }^{5}$. Similarly, in the moss Physcomitrella patens, $8.4 \%$ and $8.9 \%$ of AS events rapidly respond to PHY-mediated red and blue light perception ${ }^{6}$. Further studies showed that the interaction between PpPHY4 and a splicing factor coregulates $70 \%$ of intron retention (IR) events in response to red light in P. patens ${ }^{7}$. Moreover, light controls protein localization through PHY-mediated alternative promoter selection, allowing plants to metabolically respond to changing light conditions ${ }^{8}$. Finally, it has been extensively reported that activated PHYs induce post-translational changes in PIF proteins, including sequestration, phosphorylation, polyubiquitylation, and subsequent degradation through the $26 \mathrm{~S}$ proteasome-mediated pathway ${ }^{3}$. Although the effect of light on plant phenotypes and the plant transcriptome has been studied 
for decades ${ }^{9-11}$, the involvement of epigenetic regulatory mechanisms in light-dependent changes in the transcriptional landscape remains poorly addressed.

Posttranslational histone modifications, such as acetylation and methylation, have been associated with the induction and repression of light-responsive genes ${ }^{12,13}$. Light-dependent enrichment of the acetylation pattern of $\mathrm{H} 3$ and $\mathrm{H} 4$ in the enhancer and promoter regions of the pea plastocyanin locus PetE has been reported $^{14}$, and the hyperacetylation of the PetE promoter is linked to the transcriptional activity of this gene ${ }^{15}$. Moreover, a reduction in $\mathrm{H} 3$ acetylation is associated with a decrease in the expression of the $A$. thaliana light-responsive genes CHLOROPHYLL a/b-BINDING PROTEIN GENE (CAB2) and the RIBULOSE BISPHOSPHATE CARBOXYLASE/OXYGENASE small subunit (RBCS) ${ }^{16}$. Histone methylation regulates $\mathrm{PHY}$-mediated seed germination in A. thaliana. Upon R light illumination, photoactivated PHYB (Pfr) targets PIF1 for proteasome-mediated degradation, releasing the expression of the JUMONJI HISTONE DEMETHYLASES JMJ20 and JMJ22. As a result, JMJ20 and JMJ22 reduce the levels of H4R3me2, which leads to the activation of the gibberellic acid biosynthesis pathway to promote seed germination ${ }^{17}$.

Together with histone modification, DNA methylation is a common epigenetic mark with a direct impact on gene expression. Nevertheless, only a few reports have specifically addressed the effect of light stimuli on DNA methylation. Light-dependent nuclear organization dynamics during deetiolation are associated with a reduction in methylated DNA ${ }^{18}$. In Populus nigra, 137 genes were shown to be regulated by methylation during the day/night cycle ${ }^{19}$. Moreover, photoperiod-sensitive male sterility is regulated by RNA-directed DNA methylation (RdDM) in rice ${ }^{20}$. Finally, using a methylation-sensitive amplified polymorphism assay, DNA methylation remodelling was shown to be an active epigenetic response to different light qualities in tomato seedlings ${ }^{21}$.

Here, we investigate the impact of DNA methylation on gene expression regulation in response to PHYdependent light perception in tomato fruits. The Solanum lycopersicum genome harbours five PHYencoding genes: PHYA, PHYB1, PHYB2, PHYE and PHYF ${ }^{22}$. Previous studies have shown that PHYA, PHYB1 and PHYB2 are major regulators of fruit chloroplast maturation and nutraceutical compound accumulation ${ }^{23-26}$. In this work, the genome-wide transcriptome and methylome were comprehensively analysed in fruits from phyA and phyB1B2 null mutants, revealing that PHY-dependent DNA demethylation is a crucial stimulus for triggering ripening-associated gene expression. Moreover, our results showed that gene-body (GB) RdDM is positively correlated with gene expression during fruit development.

\section{Results}

\section{Impact of light perception impairment on the fruit transcriptome}


To investigate the role played by either PHYA or PHYB1/PHYB2 (hereafter PHYB1B2) in overall gene expression during fruit development, the transcriptome of fruits at the immature green (IG) and breaker (BK) stages from phyA and phyB1B2 null mutants as well as their wild-type (WT) counterpart, was determined by RNAsEq. Among the approximately 20,000 transcriptionally active loci in each biological replicate (Supplementary Table 1), $1.2 \%$ and $2.4 \%$ at the IG stage and $9.1 \%$ and $11.2 \%$ at the BK stage were identified as differentially expressed genes (DEGs) in phyA or phyB1B2 mutants, respectively, compared to the WT (Fig. 1a; Supplementary Table 2). For both genotypes, the number of exclusive DEGs was significantly lower in the IG stage than in the BK stage; similarly, the number of genes that were commonly regulated by PHYA and PHYB1B2 was 172 at the IG stage and 785 at the BK stage (Fig. 1b). Subsequently, the altered expression of approximately $76 \%$ (23/30) of the tested genes was validated by RT-qPCR (Supplementary Table 3). Comparison with previously reported expression data for genes involved in ripening regulation, ethylene biosynthesis and signalling, and carotenogenesis further validated our RNAseq data, as $90 \%$ of the analysed genes on average showed the expected mRNA profile at IG and BK stages. It is worth mentioning that most of the genes displayed the same transcript fluctuation in the WT, phyA and phyB1B2 genotypes, though this was somewhat attenuated in the mutants (Supplementary Table 4). These results showed that PHY-meditated light perception regulates more genes in BK than in the early stages of fruit development and that PHYB1B2 has a more substantial impact than PHYA in the fruit transcriptome in both analysed stages.

A closer look at DEGs function revealed a similar distribution of loci across MapMan categories in response to phyB1B2 and phyA mutations in both developmental stages, although with distinct abundance levels (Fig. 1C). At the IG stage, eight categories were mainly represented, including at least $2 \%$ of the DEGs identified in phyA and phyB1B2: photosynthesis, lipid metabolism, phytohormone action, RNA biosynthesis, protein modification, protein homeostasis, cell wall organization, and solute transport (Fig. 1c; Supplementary Tables 5 and 6). It is worth highlighting the abundance of the DEGs within the photosynthesis category in the phyB1B2 mutant, among which 34 out of the 37 genes were downregulated (Supplementary Table 6). In the BK stage, at least $2 \%$ of the DEGs were related to the lipid metabolism, phytohormone action, RNA biosynthesis, protein modification and homeostasis, cell wall organization and solute transport categories in both genotypes (Fig. 1c; Supplementary Tables 7 and 8). However, while phy $A$ deficiency also affected carbohydrate metabolism and external stimuli (Supplementary Table 7), the phyB1B2 mutant showed a large number of DEGs related to the cell cycle and chromatin organization (Supplementary Table 8). Interestingly, the chromatin organization category displayed $52 \mathrm{DEGs}, 45$ of which were upregulated. These genes encode nucleosome constituent histones ( $\mathrm{H} 3, \mathrm{H} 4, \mathrm{H} 2 \mathrm{~A}$ and $\mathrm{H} 2 \mathrm{~B})$; DNA methylases/demethylases; histone post-translational modifiers such as deacetylases, methylases/demethylases, histone ubiquitination factors and histone chaperones; chromatin remodelling factors; and genes involved in RNA-independent and RNA-directed DNA methylation (Supplementary Table 8). These results led us to further investigate the impact of DNA methylation on $\mathrm{PHY}$-mediated gene expression reprogramming.

\section{PHYs regulate the epigenome profile}


The global profile of methylated cytosines (mCs) in the epigenome of tomato fruits was assessed by whole-genome bisulfite sequencing in the IG and BK stages for phyA, phyB1B2 and WT genotypes. In agreement with previous reports ${ }^{27,28}$, regardless of the genotype and fruit stage, the greatest total number of mCs was located in the $\mathrm{CHH}$ context, followed by the $\mathrm{CG}$ and $\mathrm{CHG}$ contexts, while the methylation level was highest in the $\mathrm{CG}(80 \%)$ context followed by the $\mathrm{CHG}(67 \%)$ and $\mathrm{CHH}(23 \%)$ contexts (Supplementary Table 9, Supplementary Fig. 1). For further comparisons, we selected only cytosines with coverage $>10 \mathrm{X}$, and except for chromosome 9 in the transposable elements TEs enriched region, all samples met this cutoff. In all contexts, the highest cytosine density was associated with gene-rich euchromatic regions located at chromosome arm ends (Supplementary Fig. 1). Conversely, in symmetrical contexts (CG and $\mathrm{CHG}$ ), the highest methylation rates were found across pericentromeric regions enriched in TEs and in the $\mathrm{CHH}$ context in gene-rich regions associated with a higher density of sRNAs (Supplementary Fig. 1). The comparison of the methylation status between the two fruit stages showed that ripening-associated demethylation ${ }^{27}$ occurs mainly in the CG context, especially in gene-rich regions, and that it is impaired in phyB1B2 mutant BK fruits (Supplementary Fig. 1).

The subsequent comparison between genotypes revealed global epigenome alteration in phy mutants in all contexts analysed. The most remarkable observation was the presence of considerable hypermethylation in all contexts across gene-rich regions in BK-stage fruits from phyB1B2 (Fig. 2a). In contrast, phy $A$ exhibited hypermethylation in $\mathrm{CHG}$ and $\mathrm{CHH}$ contexts associated with TE-rich regions (Fig. 2a), suggesting that different PHYs control DNA methylation across specific genomic regions through distinct regulatory mechanisms. Interestingly, PHY-associated hypomethylation was exclusively detected in the CG context of gene-rich regions in IG-stage fruits from phyA and in the CHH context of TErich regions for BK-stage fruits from phyB1B2. In summary, these data revealed that both PHYA and PHYB1B2 regulate the global methylome, but PHYB1B2 has a greater impact on ripening-associated methylation reprogramming across gene-rich genomic regions in tomato fruits.

To investigate the relationship between PHY-regulated cytosine methylation and gene expression, we first identified genes with differentially methylated promoters (DMPs, $2 \mathrm{~kb}$ upstream of the transcription start site) in all three contexts. Interestingly, associated with the massive alteration previously observed, the pattern of DMPs varied with the $\mathrm{mC}$ context, stage and genotype (Fig. 2b, Supplementary Tables 10 and 11). Regarding the CG context, whereas the phyA mutant showed virtually the same frequency of hyperand hypomethylated promoters in the two stages, the status of hypermethylated promoters in phyB1B2 increased over $60 \%$ from the IG to BK stage, while the number of loci with hypomethylation decreased $50 \%$ (Fig. 2b, Supplementary Table 12). In contrast, phyA showed a greater number of hypermethylated promoters in the CHG context in the IG stage than in the BK stage, while the levels in the WT and phyB1B2 mutant remained similar upon ripening (Fig. 2b, Supplementary Table 13). In the $\mathrm{CHH}$ context, the number of hypermethylated promoters decreased in both genotypes from the IG to BK stages (Fig. 2b, Supplementary Table 14).

These results indicate that PHY deficiency results in massive promoter hypermethylation in both the IG and BK stages of tomato fruit development. Moreover, they reinforce the role of PHYB1B2 in ripening- 
associated demethylation and its putative effect on gene expression.

\section{Effect of PHY-mediated differential methylation on the transcriptome}

To assess whether the differential methylation of gene promoters affects mRNA levels, we crossed data from DEGs and DMPs between genotypes. Supplementary Fig. 2 shows scatter plots of promoter methylation vs mRNA fold changes for comparisons of the two genotypes at the two examined developmental stages in the three $\mathrm{mC}$ contexts. The most evident result was that among the thousands of loci with identified DMPs (Fig. 2b), only hundreds of the loci were also differentially expressed (Supplementary Table 15) (0.7\% for IG phyA, 1.6\% for IG phyB1B2, $5.6 \%$ for BK phyA and $7.4 \%$ for BK phyB1B2), raising an intriguing question about the biological significance of the extensive change in the methylation pattern observed in the mutants. In contrast, the percentages of the DEGs showing DMPs were $73 \%$ for IG phyA, 76\% for IG phyB1B2, 72\% for BK phyA and 75\% for BK phyB1B2 (Supplementary Fig. 2). Many more DEGs with DMPs were observed in BK than in IG fruits and in phyB1B2 than in the phyA genotype. The functional categorization of these genes revealed a similar category distribution to the DEGs (Fig. 1c, Supplementary Table 16-19). At the IG stage, there were seven categories in which at least $2 \%$ of the loci showed DMPs and differential expression in both genotypes: photosynthesis, phytohormone action, RNA biosynthesis, protein modification and homeostasis, cell wall organization and solute transport, whereas phyB1B2 additionally impacted lipid metabolism (Fig. 1c). In the BK stage, the categories in which at least $2 \%$ of the DEGs showed DMPs were lipid metabolism, phytohormone action, RNA biosynthesis, protein modification and homeostasis, cell wall organization and solute transport-related functions in both genotypes, while only phyA impacted carbohydrate metabolism and external stimuli, and only phyB1B2 affected photosynthesis, chromatin organization and cell cycle categories.

Interestingly, in the comparison of the IG and BK stages, $42.5 \%, 34.2 \%$ and $18.8 \%$ of the DMPs were associated with DEGs, while $79.5 \%, 76.6 \%$ and $71.5 \%$ of the DEGs showed differences in promoter methylation in WT, phyA and phyB1B2, respectively (Supplementary Fig. 3). All of these data demonstrated that the PHY-dependent mRNA profile is profoundly affected by promoter methylation; however, massive genome-wide PHY-induced methylation reprogramming has a still uncharacterized role beyond the regulation of mRNA accumulation. Moreover, promoter methylation has a greater effect on gene expression regulation during BK than in the IG stage. Additionally, the data showed that PHYB1B2 has a more extensive influence on gene expression regulated via promoter methylation than PHYA, reinforcing the above conclusion that PHYB1B2 affects CG ripening-associated demethylation (Supplementary Fig. 2).

\section{The sRNAome is altered by PHY deficiency}

To assess the involvement of RdDM in PHY-mediated transcriptome regulation, the sRNAome was analysed in fruits at the IG and BK stages from both mutants and the WT genotype (Supplementary Table 20a). A total of 28,314 clusters of sRNAs were identified across the whole genome in at least one of 
the samples, including 7,984 in gene bodies, 7,863 in promoter regions, 7,966 in TEs and the remaining 4,501 across intergenic regions (Supplementary Fig. 1, Supplementary Table 20b). The methylation level was evaluated for each sRNA cluster-targeted genomic region (sCTGR), and as previously observed for promoter regions, a higher proportion of hypermethylation was observed in BK fruits from phyB1B2 in the CG symmetrical context. Moreover, the greatest number of differentially methylated SCTGRs was observed in the asymmetrical context CHH. (Fig. 3a, Supplementary Table 20g-j).

sCTGR methylation levels and sRNA accumulation data were intersected, and among a total of 154, 318, 267 and 257 differentially accumulated sRNA clusters (Supplementary Table 20c-f), 88, 154, 99 and 82 also showed differential methylation changes (> 5\%) in phyA IG, phyB1B2 IG, phyA BK and phyB1B2 BK fruits, respectively (Fig. 3b, (Supplementary Table 20g-j), showing a strong association $(P<0.005)$ of the two datasets. Intriguingly, this positive association was not observed in the transition from the IG to BK stages (Supplementary Fig. 4), suggesting that the global methylation changes via RdDM could be attributed to PHY deficiency. Moreover, a clear disturbance in sRNA accumulation was observed in phyB1B2, since almost no clusters with less sRNA accumulation were observed in BK compared to the IG stage (Supplementary Fig. 4). We further analysed whether this positive correlation observed between sRNA accumulation and SCTGR methylation impacted gene expression levels. Notably, regardless of the fruit developmental stage, changes in the accumulation of sRNA located in gene bodies (GBs), and not in the promoter $(P)$ region, were positively correlated with the mRNA level (Fig. 3c, Supplementary Table 20k$\mathrm{n})$. Among these loci, two interesting examples were identified: the well-known ripening-associated genes RIPENING INHIBITOR (RIN, Solyc05g01202029) and FRUITFULL2 (FUL2, Solyc03g114830 ${ }^{30}$ ), which showed higher expression in phyB1B2 at the IG stage (Fig. 4a) and higher sRNA accumulation and SCTGR methylation across their GBs (Fig. 4b) compared to WT. The premature expression of these TFs was in agreement with the previously reported anticipation of ripening onset in the phyB1B2 mutant ${ }^{31}$. Altogether, these findings revealed (i) impaired RdDM in BK fruits of phyB1B2, indicated by the absence of clusters with less sRNA accumulation, and (ii) that GB RdDM is an important mechanism that positively regulates gene expression in a PHY-mediated manner during fruit development.

\section{PHYB1B2-dependent methylation regulates fruit chlorophyll accumulation}

The categorization of DEGs associated with differential promoter methylation revealed prominent representation of the photosynthesis category in the fruits of the phyB1B2 mutant at the IG stage (Fig. 1c). Among the 32 genes, 22 were downregulated and hypermethylated in the promoter region (Supplementary Tables 6 and 17). Most of these genes encode chlorophyll-binding proteins, structural photosystem proteins and chlorophyll biosynthetic enzymes. This might at least partly explain the reduction of $50 \%$ in the total chlorophyll level observed in phyB1B2 IG fruits (Fig. 5a). Detailed promoter analysis of the chlorophyll-related enzymes PROTOCHLOROPHYLLIDE OXIDOREDUCTASE 3 (POR3, Solyc07g054210), and two CHLOROPHYLL A/B BINDING PROTEINs (CBP, Solyc02g070990 and CAB-3C, Solyc03g005780) showed that the reduced mRNA levels of these three genes in phyB1B2 (Fig. 5b) were correlated with the presence of hypermethylated regions in the promoters. Interestingly, several of these 
hypermethylated sequences overlapped with HY5 and PIF photomorphogenic TF binding sites, such as Gbox (CACGTG), CA hybrid (GACGTA), CG hybrid (GACGTG) and PBE-box (CACATG) motifs ${ }^{32}$ (Fig. 5c). These results suggest that the transcription of genes involved in chlorophyll metabolism and the photosynthetic machinery in tomato fruits is regulated by the PHYB1B2-mediated methylation status of their promoter regions in addition to the PHY-mediated post-translational regulation of HY5 and PIF protein levels².

\section{The methylation-mediated regulation of fruit ripening is PHYB1B2-dependent}

In their seminal study, Zhong et al. (2013) ${ }^{27}$ revealed that the extensive methylation in the promoter regions of ripening-associated genes gradually decreases during fruit development. Interestingly, RNA biosynthesis, which includes transcription factors, was the most abundant functional category among the DEGs that showed DMPs (Fig. 1C). Thus, we examined a set of ripening-associated master transcription factors: RIN, Solyc05g012020, NON-RIPENING (NOR, Solyc10g00688033), COLORLESS NORIPENING (CNR, Solyc02g077920 $\left.{ }^{34}\right)$ and APETALA2a (AP2a, Solyc03g044300 $\left.{ }^{35}\right)$. The evaluation of the promoter regions clearly showed that while their methylation level decreased from the IG to BK stage in the WT genotype, they remained highly methylated in phyB1B2 (Fig. 6a). As previously observed, several hypermethylated regions are closely linked to HY5 and PIF binding sites. The maintenance of high methylation levels in the promoters of these key regulatory genes at the onset of fruit ripening was highly correlated with their transcriptional downregulation at the BK stage (Fig. 6b).

Carotenoid accumulation is probably the most appealing and best investigated trait of tomato fruits; in agreement with previous findings ${ }^{26}$, ripe phyB1B2 fruits showed a five-fold reduction in carotenoid content compared to WT (Fig. 7a).

With the aim of evaluating whether this effect is a consequence of the methylation-mediated regulation of carotenoid biosynthesis genes, we further analysed the promoters of PHYTOENE SYNTHASE 1 (PSY1, Solyc03g031860), PHYTOENE DESATURASE (PDS, Solyc03g123760), 15-CIS- Z-CAROTENE (ZISO, Solyc12g098710) and ZETA-CAROTENE DESATURASE (ZDS, Solyc01g097810), which, with the exception of $P D S$, were hypermethylated in phyB1B2 BK fruits (Supplementary Table 11). The mC profile confirmed the presence of hypermethylated regions in all four promoters, which were predominantly located near light-dependent TF binding motifs in the PSY1, PDS and ZISO promoters (Fig. 7b), explaining the reduced mRNA levels of these genes observed in phyB1B2 (Fig. 7c).

RIN is one of the main TFs controlling ripening-associated genes by directly binding to their promoters. RIN binding occurs in concert with the demethylation of its targets ${ }^{27}$. To examine whether RIN binding site methylation could be affected by the phyB1B2 mutation in the ripening-related master transcription factors and carotenoid biosynthesis gene promoters, we mapped the available RIN ChIP-seq data ${ }^{27}$ and performed de novo motif discovery (Supplementary Fig. 5). Interestingly, the levels of $\mathrm{mCs}$ associated with RIN targets were higher in the NOR, CNR and AP2a promoters in phyB1B2 than in WT. Moreover, the 
$R I N$ promoter itself was hypermethylated across the RIN binding site in phyB1B2 BK fruits, suggesting a positive feedback regulatory mechanism (Fig. 6a). Finally, in the phyB1B2 mutant, the PSY1, PDS, ZISO and ZDS promoters showed higher methylation overlapping with RIN target binding motifs, indicating that the upregulation of carotenoid biosynthesis genes during tomato ripening is dependent on the PHYB1B2mediated demethylation of RIN target sites.

Altogether, our findings showed that PHYB1B2 is a major player in fruit ripening by controlling the promoter demethylation of master transcriptional regulators and carotenoid biosynthesis genes.

\section{Discussion}

The dynamic methylation pattern during tomato fruit development has been demonstrated to be a critical ripening regulation mechanism ${ }^{27,28}$. DNA demethylation, mainly in the CG context, triggers the activation of genes involved in ripening and is required for pigment accumulation and ethylene synthesis 27,36 . Simultaneously, the dynamic epigenome during fruit development is strictly regulated by environmental cues $^{37}$. The prevailing model establishes PHYs as major components involved in the coordination of fruit physiology with the ever-changing light and temperature environment ${ }^{23,26}$. Thus, we explored the link between fruit epigenome reprogramming and these well-established light and temperature sensors.

Our data clearly showed that phyA and phyB1B2 deficiencies modified the epigenome profile through methylome and sRNAome reprogramming. In particular, PHY-mediated DMPs and GB methylation impacted the transcriptome pattern, affecting tomato fruit development and demonstrating that a portion of the ripening-associated demethylation previously reported ${ }^{27}$ is dependent on active PHYs, especially PHYB1B2. However, the massive alteration of methylation patterns observed in PHY mutants suggests the existence of a still unclear genome regulatory mechanism. Whether it is related to light-induced $\mathrm{AS}^{3}$ and/or PHY-mediated alternative promoter usage to control protein localization ${ }^{8}$, as mentioned above, remains to be explored.

The phyA and phyB1B2 mutants showed a positive correlation between cluster sRNA accumulation, target methylation in GB and mRNA levels. In angiosperms, GB methylation has been associated with constitutively expressed genes ${ }^{38,39}$; however, $\mathrm{PHY}$ deficiency intriguingly seems to deregulate this mechanism, affecting temporally and spatially regulated genes. The RIN and FUL2 examples analysed here clearly showed that SRNA accumulation and methylation were mainly located near transposable elements (TEs) (Fig. 4). It is known that the insertion of TEs within GB can disrupt gene expression; thus, methylation-mediated TE silencing and GB methylation are evolutionarily linked ${ }^{38}$. The enhancement of TE-associated DNA methylation in GB (Fig. 3c) and the absence of clusters with less sRNA accumulation in BK compared to the IG stage in phyB1B2 (Supplementary Fig. 4) can be explained by the overexpression of canonical RdDM genes. Solyc12g008420 and Solyc06g050510 encode homologs of RNA-DEPENDENT RNA POLYMERASE (RDRP) and the associated factor SNF2 DOMAIN-CONTAINING PROTEIN CLASSY 1 (CLSY1), respectively, both of which were upregulated in BK fruits from phyB1B2 
plants (Supplementary Table 2). These proteins are key players in canonical RdDM in $A$. thaliana required for PolIV-dependent sRNA production ${ }^{40}$. Similarly, Solyc09g082480 and Solyc03g083170, which were also upregulated in phyB1B2 BK fruits, are homologs of $A$. thaliana RNA-DIRECTED DNA METHYLATION 1 (RDM1) and DEFECTIVE IN MERISTEM SILENCING 3 (DMS3), respectively. The protein products of these genes, together with DEFECTIVE IN RNA- DIRECTED DNA METHYLATION 1 (DRD1), form the DDR complex, which enables RNA Pol V transcription ${ }^{41}$. To our knowledge, this is the first report to associate PHY-mediated sRNA accumulation and DNA methylation with mRNA levels in plants.

Several pieces of evidence have shown that PHYB1B2 has a more substantial impact on genome regulation than PHYA. For example, BK fruits from phyB1B2 displayed (i) a large number of DEGs associated with chromatin organization (Fig. 1C); (ii) overall promoter hypermethylation in the CG context (Fig. 2b); (iii) the highest number of DEGs associated with DMPs (Supplementary Fig. 2); and (iv) half the number of DMPs associated with DEGs between the IG and BK stages compared to the WT (Supplementary Fig. 3). These massive epigenomic alterations in phyB1B2 led us to look more closely at genes related to chromatin organization that showed alterations in mRNA levels.

The chromomethylase SIMET1L (Solyc01g006100) (also referred to as SICMT3 ${ }^{42}$ ) displays the highest transcript abundance in immature fruits, which declines towards the fully ripe stage ${ }^{43}$. Hence, in line with the higher level of DNA methylation, our transcriptome analysis showed that SIMET1L was upregulated in phyB1B2 BK fruits. Conversely, the upregulation of the SIROS1L demethylase (Solyc09g00908043; also referred to as $S I D M L 1^{44}$ ) was also observed in phyB1B2 BK fruits, which might seem contradictory at first glance. However, it has been reported that the Arabidopsis thaliana ROS1 gene promoter contains a DNA methylation monitoring sequence (MEMS) associated with a Helitron transposon, which is methylated by AtMET1, positively regulating AtROS1 gene expression ${ }^{45}$. Similarly, SIROS1L harbours two transposable elements within its promoter and showed a higher methylation level in phyB1B2 than in the WT genotype, suggesting a similar regulatory mechanism in tomato (Supplementary Fig. 6, Supplementary Table 15).

The tomato homologue of $A$. thaliana DECREASED DNA METHYLATION 1 (DDM1, Solyc02g085390) showed higher mRNA expression in phyB1B2 mutant BK fruits than in their WT counterparts. DDM1 is a chromatin remodelling protein required for maintaining DNA methylation in the symmetric cytosine sequence ${ }^{46}$, which can be associated with the observed methylation differences in the CG context in phyB1B2 (Fig. 2a).

Several histone modifiers showed altered expression in BK fruits from the phyB1B2 mutant (Supplementary Table 8). The methylation of lysine residues 9 and 27 on histone H3 is associated with repressed genes. Histone lysine methyltransferases are classified into five groups based on their domain architecture and/or differences in enzymatic activity ${ }^{47}$. The BK fruits of the phyB1B2 mutant displayed three differentially expressed lysine methyltransferases: Solyc03g082860, an upregulated H3K27 Class IV homologue, and Solyc06g008130 and Solyc06g083760, two H3K9 Class V homologs that showed lower and higher expression than in WT fruits, respectively. Histone arginine methylation is catalysed by a 
family of enzymes known as protein arginine methyltransferases (PRMTs). Solyc12g099560, a PRMT4a/b homologue, was upregulated in phyB1B2 BK fruits. Interestingly, in A. thaliana, PRMT4s modulate key regulatory genes associated with the light response ${ }^{48}$, reinforcing the link between the PHYB1B2 photoreceptor and epigenetic control. Finally, tomato histone demethylases have been recently identified. S/JMJ6, whose expression peaks immediately after the BK stage, has been characterized as a positive regulator of fruit ripening by removing the H3K27 methylation of ripening-related genes, and SIJMJ6-overexpressing lines show increased carotenoid levels ${ }^{49}$. SIJMJC1 (Solyc01g006680), which exhibits the same expression pattern ${ }^{49}$, is downregulated in the phyB1B2 mutant, suggesting that this gene might exhibit a similar regulatory function to its paralogue, inducing ripening in a PHYB1B2dependent manner (Figs. 6 and 7).

Histone deacetylation plays a crucial role in the regulation of eukaryotic gene activity and is associated with inactive chromatin ${ }^{40}$. Histone deacetylation is catalysed by histone deacetylases (HDACs). Fifteen HDACs were identified in the tomato genome ${ }^{50}$. Among these HDACs, SIHDA10 (Solyc01g009120) and SIHDT3 (Solyc11g066840) were found to be downregulated and upregulated in phyB1B2 BK fruits, respectively. SIHDA10 is localized in the chloroplast, and its transcript is highly expressed in photosynthetic tissues ${ }^{50}$; whether SIHDA10 deacetylates chloroplast proteins by silencing photosynthesis-related genes remains to be determined. Although SIHDT3 is mainly expressed in immature stages of fruit development and its expression declines with ripening, its silencing results in delayed ripening and reduced RIN expression and carotenogenesis. On the other hand, the expression level of SIHDT3 is increased in ripening-deficient mutants such as $\mathrm{Nr}$ or $\mathrm{rin}^{51}$. Our results showed that phyB1B2 mutant fruits displayed higher expression of SIHDT3 and reduced RIN transcript levels at the BK stage, suggesting reciprocal regulation between these two factors (Fig. 8a). During the IG stage, SIHDT3 is highly expressed, contributing to the epigenetic inhibition of ripening. The reduction in SIHDT3 expression towards BK releases DNA methylation and both directly and indirectly upregulates RIN. Additionally, PHYB1B2 inhibits the expression of SIHDT3 and induces that of RIN, thereby inducing or repressing DNA methylation, respectively. Finally, RIN maintains SIHDT3 downregulation in BK ${ }^{51}$. This mechanism precisely tunes ripening-related epigenetic reprogramming and contributes to explaining the high methylation levels observed in the phyB1B2 mutant (Fig. 2).

The link between DNA methylation levels and tomato fruit ripening-associated gene expression has been previously reported ${ }^{27,28}$, but the stimuli and the molecular mechanisms underlying this relationship remained unknown. The integrated analysis of the experimental evidence together with previous gene functional studies in tomato and $A$. thaliana allowed us to propose that PHYB1B2 is an important triggering factor for chromatin remodelling and, consequently, transcriptional regulation during fruit development. PHY signal transduction is mediated by the coordinated expression of DNA methylases/demethylases, histone-modifying enzymes and chromatin remodelling factors resulting in the induction of photosynthesis and ripening-related genes in immature and breaker fruit stages, respectively (Fig. 8b). The vast reservoir of data released here brings a new level of understanding about 
how epigenetic mechanisms orchestrate the response to light and temperature fluctuations affecting important agronomical traits in fleshy fruits.

\section{Material And Methods}

\section{Plant material, growth conditions and sampling}

phyA and phyB1B2 phytochrome mutants in the Solanum lycopersicum (cv. MoneyMaker) genetic background were previously characterized ${ }^{52-54}$. Tomato seeds were grown in $9 \mathrm{~L}$ pots containing a 1:1 mixture of commercial substrate and expanded vermiculite, supplemented with $1 \mathrm{~g} \mathrm{~L}^{-1}$ of NPK 10:10:10, $4 \mathrm{~g} \mathrm{~L}^{-1}$ of dolomite limestone $\left(\mathrm{MgCO}_{3}+\mathrm{CaCO}_{3}\right)$ and $2 \mathrm{~g} \mathrm{~L}^{-1}$ thermophosphate at $24 / 18^{\circ} \mathrm{C}$ under a $16 / 8 \mathrm{~h}$ light/dark cycle under $230-250 \mu \mathrm{mol}$ photons $\mathrm{m}^{-2} \mathrm{~s}^{-1}$ irradiation and a relative humidity of $55 \%$. Five replicates per genotype were cultivated. Fruits were sampled at the immature green $(15 \mathrm{~mm}$ diameter), mature green (when the placenta displays a gelatinous aspect), breaker (beginning of ripening process when the fruit shows the first yellowish colouration) and red ripe (7 days after the breaker stage) stages. All fruits were harvested at the same time of day with four biological replicates (each replicate was composed of a single fruit per plant). The columella, placenta, and seeds were immediately removed, and the remaining tissues were frozen in liquid nitrogen, ground and freeze-dried for subsequent analysis.

\section{Transcriptional profile}

Total RNA was extracted from immature green and breaker stage fruits with three independent biological replicates of each genotype using a Promega ReliaPrep RNA tissue kit according to the manufacturer's instructions. The RNA concentration was determined with a spectrophotometer (Nanodrop ND-1000; NanoDrop Technologies, Wilmington, DE, U.S.A.), RNA quality was assessed with a BioAnalyzer 2100 (Agilent Technologies), and RNA libraries were constructed following the recommendations of an Illumina Kit (Directional mRNA-Seq Sample Preparation) and sequenced using the Illumina NovaSeq 6000 System. Each library was sequenced, generating approximately 20 million 150 bp paired end reads per sample. The raw sequencing reads that were generated were analysed with FastQC (http://www.bioinformatics.babraham.ac.uk/projects/fastqc/) and were filtered and cleaned using Trimmomatic ${ }^{55}$ (Parameters: ILLUMINACLIP: TruSeq3-PE.fa:2:30:10LEADING:3 TRAILING:3 SLIDINGWINDOW:4:20 MINLEN:50). At least 95\% (19.1-27.9 M ) of the reads met the quality criteria and were mapped to the tomato reference genome sequence SL3.0 with the ITAG3.2 annotation using STAR v2.4.2. allowing one mismatch ${ }^{56}$, approximately $84 \%$ of the reads were uniquely mapped (Supplementary Table 1) and were used for statistical analysis.

\section{Reverse transcription quantitative PCR (RT-qPCR)}

Total RNA extraction was performed with the ReliaPrep ${ }^{T M}$ RNA Cell and Tissue Miniprep System (Promega), and cDNA synthesis was conducted with SuperScript ${ }^{\mathrm{TM}}$ IV Reverse Transcriptase (Invitrogen). The primers used for qPCR are listed in Supplementary Table 21. RT-qPCR was performed in a QStudio6 - A1769 PCR Real-Time thermocycler using 2X Power SYBR Green Master Mix in a final volume of $10 \mu \mathrm{L}$. 
Absolute fluorescence data were analysed using LinRegPCR software to obtain Ct and primer efficiency values. Relative mRNA abundance was calculated and normalized according to the $\triangle \triangle \mathrm{Ct}$ method using EXPRESSED and CAC as reference genes ${ }^{57}$.

\section{MethylC-Seq analysis}

Methyl C sequencing was performed as described in a previous report ${ }^{58}$. gDNA $(\sim 5 \mathrm{~g})$ was extracted from a pool of the same three biological replicates used in the transcriptome analyses, obtained from three IG and BK fruit samples per genotype, using the DNeasy Plant maxi kit (Qiagen). The libraries were prepared with the EZ DNA Methylation-Gold Kit (Zymo Research) and the Accel-NGS ${ }^{\circledR}$ Methyl-Seq DNA Library Kit (Swift Biosciences) and further sequenced using the Illumina NovaSeq 6000 platform. Over $240 \mathrm{M}$ reads were sequenced from each genotype and stage. Raw reads were screened for quality using Trimmomatic $^{55}$ (parameters: ILLUMINACLIP:TruSeq3-PE.fa:2:30:10 LEADING:3 TRAILING:3 SLIDINGWINDOW:4:20 MINLEN:50). Mapping to the tomato reference genome sequence SL3.0 and the assessment of global methylation status were performed using Bismark ${ }^{59}$ (parameters: bismark -q bowtie2 -non_directional -N 1 -p 4), and the methylation status of DNA in the three possible contexts (CG, $\mathrm{CHG}$ and $\mathrm{CHH}$ ) was distinguished. At least $130 \mathrm{M}$ reads were uniquely mapped (Supplementary Table 9). The Bioconductor package methylKit ${ }^{60}$ was used for the detection of methylation levels across the analysed regions: promoters (2 kb upstream of transcription start site) and sRNA cluster-targeted genome regions (sCTGRs). Only Cs with 10X coverage were considered. Methylation differences with an FDR< 0.05 in each comparison (WT vs phyA; WT vs phyB1B2) were recorded as differentially methylated promoters (DMPs) or differentially methylated sCTGRs. Differential methylation in the CG, CHG and/or $\mathrm{CHH}$ context was considered to exist if the region contained at least 10 differentially methylated $\mathrm{Cs}$ in the corresponding context. Finally, for the comparison of global methylation levels between genotypes, only common Cs with at least $10 \mathrm{X}$ coverage in all samples were analysed.

\section{sRNAome profile}

sRNA extraction and quality parameters were determined from three independent biological replicates of green and breaker stage fruits from each genotype, as described above in the "Transcriptional profile" section. After RNA integrity confirmation, libraries were prepared using a TruSeq Small RNA Library Prep and sequenced using the Illumina HiSeq 4000 platform to generate a read length of $50 \mathrm{bp}$. The raw sequencing reads that were generated were quality trimmed with Trimmomatic ${ }^{55}$ to retain reads of $18-24$ nt in length (parameters: ILLUMINACLIP:TruSeq3-SE:2:30:10 LEADING:3 TRAILING:3

SLIDINGWINDOW:4:15 MINLEN:18 AVGQUAL:25). A minimum of 38\% (WT/breaker/A) and a maximum of $85 \%$ (WT/immature green/A) of the reads achieved the quality criteria and were used for further analyses (Supplementary Table 20a). All libraries were aligned to genome version SL3.0 using ShortStack v3.8.1 ${ }^{61}$ with default parameters (allowing the distribution of multimapping reads according to the local genomic context). Then, the de novo identification of clusters of sRNAs was performed for all libraries, and individual counts for each library and cluster were obtained using the same software.

\section{Statistical analysis for RNAseq and sRNAome}


Genes/sRNA clusters with read/count numbers smaller than two per million were removed. Read/count values were normalized according to the library size factors. Statistical analyses were performed with edgeR from Bioconductor ${ }^{62,63}$ using a genewise negative binomial generalized linear model with the quasi-likelihood test ${ }^{64}$ and a cutoff of the false discovery rate (FDR) $\leq 0.05$.

\section{Gene functional categorization}

The DEGs were functionally categorized with MapMan application software ${ }^{65}$ followed by hand-curated annotation using MapMan categories.

In silico regulatory motif predictions and RIN ChIP-seq analyses

To predict the transcription factor binding motifs, we used the complete collection of 530 plant transcription factor-binding sites (TFBS) modelled as position frequency matrices (PFMs) from the JASPAR 2020 database ${ }^{66}$. Putative binding sites were obtained by scanning the whole S. lycopersicum 3.00 genome with Fimo ${ }^{67}$, p-value $<1 e-5$.

RIN ChIPseq reads were downloaded from the Sequence Read Archive (SRA) (accession SRX15083 ${ }^{27}$ ) and mapped to tomato genome version SL3.0 with STAR ${ }^{56}$ (version 2.7.3X, parameters:

outFilterMismatchNmax 3, alignEndsType EndToEnd, alignIntronMax 5), and peak calling was performed by using Macs2 ${ }^{68}$ (version 2.2.7.1, default parameters). Regions of 200 bp centred on the top-scoring peaks (score > 100, $n=327$ ) among transposable elements were retrieved, and the binding motif was inferred de novo by using the MEME algorithm (Supplemental Fig. 5) 67.

\section{Carotenoid and chlorophyll analysis}

Carotenoid and chlorophyll extraction was performed from aliquots of $20 \mathrm{mg}$ dry mass, sequentially with $100 \mu \mathrm{L}$ of a saturated solution of $\mathrm{NaCl}, 200 \mu \mathrm{L}$ of dichloromethane and $500 \mu \mathrm{L}$ of hexane:diethyl ether $1: 1$ $(\mathrm{v} / \mathrm{v})$. The pellet was extracted three additional times with $500 \mu \mathrm{L}$ of $1: 1(\mathrm{v} / \mathrm{v})$ hexane:diethyl ether. All supernatant fractions were combined, completely dried by vacuum and suspended in $200 \mu \mathrm{L}$ of acetonitrile. Chlorophyll, phytoene, phytofluene, lycopene, $\beta$-carotene and lutein levels were determined via HPLC with a photodiode array detector ${ }^{69}$.

\section{Statistical analysis of RT-qPCR and metabolites}

Statistical analyses of the RT-qPCR (Student's t-test, $p \leq 0,05$ ) and metabolic data (ANOVA, Tukey's test. $p$ $\leq 0,05)$ were performed with InfoStat/F software (http://www.infostat.com.ar).

\section{Declarations}

\section{Competing interests}

The authors declare no competing interests. 


\section{Author contributions}

RB performed most of the experiments and analysed the data; LB, NB, LAH and RZ analysed the data; DR performed the experiments; RB, LF, MR and LB conceived the project, designed the experiments and wrote the paper, which was revised and approved by all authors. LB agrees to serve as the author responsible for contact and ensures communication.

\section{ACKNOWLEDGMENTS}

This work was supported by FAPESP (Fundação de Amparo à Pesquisa do Estado de São Paulo, Grant Number \#2016/01128-9); RB was a recipient of a FAPESP fellowship (\#2017/24354-7). MR was a recipient of a CNPq fellowship. LB was a recipient of a CAPES-PRINT scholarship (88887.370243/2019$00)$.

\section{Data availability}

All high-throughput sequencing data reported in this paper have been uploaded to the Sequence Read Archive (SRA) under NCBI Bioproject PRJNA646733, with accession numbers SUB7763724, SUB7782168 and SUB7791358 for RNAseq, WGBS and small RNAseq, respectively.

\section{References}

1. Kaiserli, E., Perrella, G. \& Davidson, M. L. Light and temperature shape nuclear architecture and gene expression. Curr. Opin. Plant Biol. 45, 103-111 (2018).

2. Galvão, V. C. \& Fankhauser, C. Sensing the light environment in plants: photoreceptors and early signaling steps. Curr. Opin. Neurobiol. 34, 46-53 (2015).

3. Paik, I. \& Huq, E. Plant photoreceptors: Multi-functional sensory proteins and their signaling networks. Semin. Cell Dev. Biol. 92, 114-121 (2019).

4. Cheng, Y.-L. \& Tu, S.-L. Alternative Splicing and Cross-Talk with Light Signaling. Plant Cell Physiol. 59, 1104-1110 (2018).

5. Shikata, H. et al. Phytochrome controls alternative splicing to mediate light responses in Arabidopsis. Proc. Natl. Acad. Sci. 111, 18781-18786 (2014).

6. Wu, H.-P. et al. Genome-wide analysis of light-regulated alternative splicing mediated by photoreceptors in Physcomitrella patens. Genome Biol. 15, R10 (2014).

7. Lin, B.-Y., Shih, C.-J., Hsieh, H.-Y., Chen, H.-C. \& Tu, S.-L. Phytochrome Coordinates with a hnRNP to Regulate Alternative Splicing via an Exonic Splicing Silencer. Plant Physiol. 182, 243 LP - 254 (2020).

8. Ushijima, T. et al. Light Controls Protein Localization through Phytochrome-Mediated Alternative Promoter Selection. Cell 171, 1316-1325.e12 (2017). 
9. Mazzella, M. A. et al. Phytochrome control of the Arabidopsis transcriptome anticipates seedling exposure to light. Plant Cell 17, 2507-2516 (2005).

10. Ibarra, S. E., Auge, G., Sánchez, R. A. \& Botto, J. F. Transcriptional programs related to phytochrome a function in arabidopsis seed germination. Mol. Plant 6, 1261-1273 (2013).

11. Carlson, K. D., Bhogale, S., Anderson, D., Tomanek, L. \& Madlung, A. Phytochrome a regulates carbon flux in dark grown tomato seedlings. Front. Plant Sci. 10, 1-20 (2019).

12. Perrella, G. \& Kaiserli, E. Light behind the curtain: photoregulation of nuclear architecture and chromatin dynamics in plants. New Phytol. 212, 908-919 (2016).

13. Tessadori, F. et al. Phytochrome B and histone deacetylase 6 control light-induced chromatin compaction in Arabidopsis thaliana. PLoS Genet. 5, e1000638 (2009).

14. Chua, Y. L., Brown, A. P. \& Gray, J. C. Targeted histone acetylation and altered nuclease accessibility over short regions of the pea plastocyanin gene. Plant Cell 13, 599-612 (2001).

15. Chua, Y. L., Watson, L. A. \& Gray, J. C. The transcriptional enhancer of the pea plastocyanin gene associates with the nuclear matrix and regulates gene expression through histone acetylation. Plant Cell 15, 1468-1479 (2003).

16. Bertrand, C. et al. Arabidopsis HAF2 gene encoding TATA-binding protein (TBP)-associated factor TAF1, is required to integrate light signals to regulate gene expression and growth. J. Biol. Chem. 280, 1465-1473 (2005).

17. Cho, J.-N. et al. Control of seed germination by light-induced histone arginine demethylation activity. Dev. Cell 22, 736-748 (2012).

18. Bourbousse, C., Barneche, F. \& Laloi, C. Plant Chromatin Catches the Sun. Frontiers in Plant Science 10,1728 (2020).

19. Ding, C. J., Liang, L. X., Diao, S., Su, X. H. \& Zhang, B. Y. Genome-wide analysis of day/night DNA methylation differences in Populus nigra. PLoS One 13, 1-14 (2018).

20. Ding, J. et al. RNA-directed DNA methylation is involved in regulating photoperiod-sensitive male sterility in rice. Mol. Plant 5, 1210-1216 (2012).

21. Omidvar, V. \& Fellner, M. DNA Methylation and Transcriptomic Changes in Response to Different Lights and Stresses in 7B-1 Male-Sterile Tomato. PLoS One 10, e0121864 (2015).

22. Alba, R., Kelmenson, P. M., Cordonnier-Pratt, M.-M. \& Pratt, L. H. The Phytochrome Gene Family in Tomato and the Rapid Differential Evolution of this Family in Angiosperms. Mol. Biol. Evol. 17, 362$373(2000)$.

23. Alves, F. R. R. et al. Beyond the limits of photoperception: constitutively active PHYTOCHROME B2 overexpression as a means of improving fruit nutritional quality in tomato. Plant Biotechnol. J. n/a, (2020).

24. Gramegna, G. et al. PHYTOCHROME-INTERACTING FACTOR 3 mediates light-dependent induction of tocopherol biosynthesis during tomato fruit ripening. Plant. Cell Environ. 42, 1328-1339 (2019). 
25. Bianchetti, R. et al. Fruit-localized phytochromes regulate plastid biogenesis, starch synthesis, and carotenoid metabolism in tomato. J. Exp. Bot. 69, 3573-3586 (2018).

26. Bianchetti, R. et al. Phytochrome-Dependent Temperature Perception Modulates Isoprenoid Metabolism. Plant Physiol. 183, 869 LP - 882 (2020).

27. Zhong, S. et al. Single-base resolution methylomes of tomato fruit development reveal epigenome modifications associated with ripening. Nat. Biotechnol. 31, 154-159 (2013).

28. Zuo, J. et al. Relationships between genome methylation, levels of non-coding RNAs, mRNAs and metabolites in ripening tomato fruit. Plant J. (2020). doi:10.1111/tpj.14778

29. Vrebalov, J. et al. A MADS-Box Gene Necessary for Fruit Ripening at the Tomato Ripening-Inhibitor Locus. Science (80). 296, 343 LP - 346 (2002).

30. Bemer, M. et al. The Tomato FRUITFULL Homologs TDR4/FUL1 and MBP7/FUL2 Regulate EthyleneIndependent Aspects of Fruit Ripening. Plant Cel/ 24, 4437 LP - 4451 (2012).

31. Gupta, S. K. et al. Complex and shifting interactions of phytochromes regulate fruit development in tomato. Plant. Cell Environ. 37, 1688-1702 (2014).

32. Zhang, Y. et al. A Quartet of PIF bHLH Factors Provides a Transcriptionally Centered Signaling Hub That Regulates Seedling Morphogenesis through Differential Expression-Patterning of Shared Target Genes in Arabidopsis. PLOS Genet. 9, e1003244 (2013).

33. Giovannoni, J. J. Fruit ripening mutants yield insights into ripening control. Curr. Opin. Plant Biol. 10, 283-289 (2007).

34. Manning, K. et al. A naturally occurring epigenetic mutation in a gene encoding an SBP-box transcription factor inhibits tomato fruit ripening. Nat. Genet. 38, 948-952 (2006).

35. Karlova, R. et al. Transcriptome and Metabolite Profiling Show That APETALA2a Is a Major Regulator of Tomato Fruit Ripening. Plant Cell 23, 923 LP - 941 (2011).

36. Lang, Z. et al. Critical roles of DNA demethylation in the activation of ripening-induced genes and inhibition of ripening-repressed genes in tomato fruit. Proc. Natl. Acad. Sci. 114, E4511-E4519 (2017).

37. Zhang, B. et al. Chilling-induced tomato flavor loss is associated with altered volatile synthesis and transient changes in DNA methylation. Proc. Natl. Acad. Sci. 113, 12580 LP - 12585 (2016).

38. Bewick, A. J. \& Schmitz, R. J. Gene body DNA methylation in plants. Curr. Opin. Plant Biol. 36, 103110 (2017).

39. Lu, X. et al. Genome-Wide Epigenetic Regulation of Gene Transcription in Maize Seeds. PLoS One 10, e0139582 (2015).

40. Zhang, H., Lang, Z. \& Zhu, J. K. Dynamics and function of DNA methylation in plants. Nat. Rev. Mol. Cell Biol. 19, 489-506 (2018).

41. Pikaard, C. S. \& Scheid, O. M. Epigenetic regulation in plants. Cold Spring Harb. Perspect. Biol. 6, 132 (2014). 
42. Gallusci, P., Hodgman, C., Teyssier, E. \& Seymour, G. B. DNA Methylation and Chromatin Regulation during Fleshy Fruit Development and Ripening. Frontiers in Plant Science 7, 807 (2016).

43. Cao, D. et al. Genome-wide identification of cytosine-5 DNA methyltransferases and demethylases in Solanum lycopersicum. Gene 550, 230-237 (2014).

44. Liu, R. et al. A DEMETER-like DNA demethylase governs tomato fruit ripening. Proc. Natl. Acad. Sci. 112, 10804 LP - 10809 (2015).

45. Lei, M. et al. Regulatory link between DNA methylation and active demethylation in Arabidopsis. Proc. Natl. Acad. Sci. 112, 3553 LP - 3557 (2015).

46. Zemach, A. et al. The arabidopsis nucleosome remodeler DDM1 allows DNA methyltransferases to access H1-containing heterochromatin. Cell 153, 193-205 (2013).

47. Pontvianne, F., Blevins, T. \& Pikaard, C. S. Arabidopsis Histone Lysine Methyltransferases. Adv Bot Res. 53, 1-22 (2010).

48. Hernando, C. E., Sanchez, S. E., Mancini, E. \& Yanovsky, M. J. Genome wide comparative analysis of the effects of PRMT5 and PRMT4/CARM1 arginine methyltransferases on the Arabidopsis thaliana transcriptome. BMC Genomics 16, 192 (2015).

49. Li, Z. et al. Histone demethylase SIJMJ6 promotes fruit ripening by removing H3K27 methylation of ripening-related genes in tomato. New Phytol. 227, 1138-1156 (2020).

50. Zhao, L. et al. Identification and characterization of histone deacetylases in tomato (Solanum lycopersicum). Frontiers in Plant Science 5, 760 (2015).

51. Guo, J. E. et al. Silencing of histone deacetylase SIHDT3 delays fruit ripening and suppresses carotenoid accumulation in tomato. Plant Sci. 265, 29-38 (2017).

52. Kerckhoffs, L. H. J. et al. Molecular analysis of tri-mutant alleles in tomato indicates the Tri locus is the gene encoding the apoprotein of phytochrome B1. Planta 199, 152-157 (1996).

53. Lazarova, G. I. et al. Molecular analysis of PHYA in wild-type and phytochrome A-deficient mutants of tomato. Plant J. 14, 653-662 (1998).

54. Kerckhoffs, L. H. J. et al. Characterization of the gene encoding the apoprotein of phytochrome B2 in tomato, and identification of molecular lesions in two mutant alleles. Mol. Gen. Genet. 261, 901-907 (1999).

55. Bolger, A. M., Lohse, M. \& Usadel, B. Trimmomatic: A flexible trimmer for lllumina sequence data. Bioinformatics 30, 2114-2120 (2014).

56. Dobin, A. et al. STAR: ultrafast universal RNA-seq aligner. Bioinformatics 29, 15-21 (2013).

57. Expósito-Rodríguez, M., Borges, A. A., Borges-Pérez, A. \& Pérez, J. A. Selection of internal control genes for quantitative real-time RT-PCR studies during tomato development process. BMC Plant Biol. 8, 131 (2008).

58. Lister, R. et al. Highly Integrated Single-Base Resolution Maps of the Epigenome in Arabidopsis. Cell 133, 523-536 (2008). 
59. Krueger, F. \& Andrews, S. R. Bismark: A flexible aligner and methylation caller for Bisulfite-Seq applications. Bioinformatics 27, 1571-1572 (2011).

60. Akalin, A. et al. MethylKit: a comprehensive R package for the analysis of genome-wide DNA methylation profiles. Genome Biol. 13, R87 (2012).

61. Axtell, M. J. ShortStack: Comprehensive annotation and quantification of small RNA genes. Rna 19, 740-751 (2013).

62. Robinson, M. D., McCarthy, D. J. \& Smyth, G. K. edgeR: a Bioconductor package for differential expression analysis of digital gene expression data. Bioinformatics 26, 139-140 (2009).

63. McCarthy, D. J., Chen, Y. \& Smyth, G. K. Differential expression analysis of multifactor RNA-Seq experiments with respect to biological variation. Nucleic Acids Res. 40, 4288-4297 (2012).

64. Chen, Y., Lun, A. T. L. \& Smyth, G. K. From reads to genes to pathways: differential expression analysis of RNA-Seq experiments using Rsubread and the edgeR quasi-likelihood pipeline. F1000 Research 5, 1438 (2016).

65. Thimm, O. et al. MapMan: a user-driven tool to display genomics data sets onto diagrams of metabolic pathways and other biological processes. Plant J. 37, 914-939 (2004).

66. Fornes, O. et al. JASPAR 2020: update of the open-access database of transcription factor binding profiles. Nucleic Acids Res. 48, D87-D92 (2019).

67. Bailey, T. L., Johnson, J., Grant, C. E. \& Noble, W. S. The MEME Suite. Nucleic Acids Res. 43, W39W49 (2015).

68. Zhang, Y. et al. Model-based Analysis of ChIP-Seq (MACS). Genome Biol. 9, R137 (2008).

69. Lira, B. S. et al. Manipulation of a senescence-associated gene improves fleshy fruit yield. Plant Physiol. 175, 77-91 (2017).

\section{Figures}




\section{a}

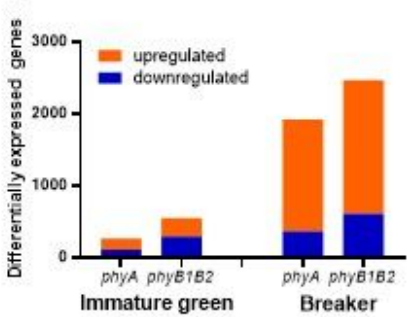

C

IG

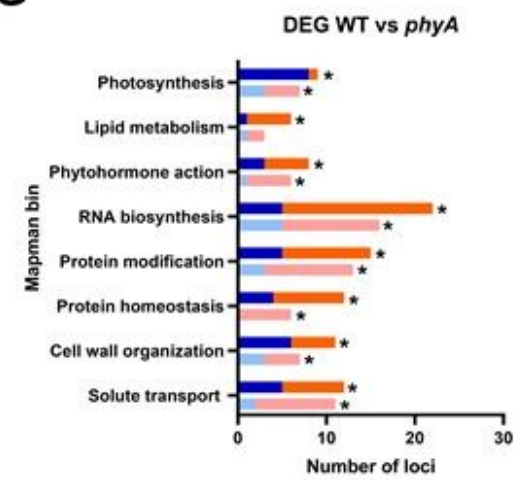

\section{BK}

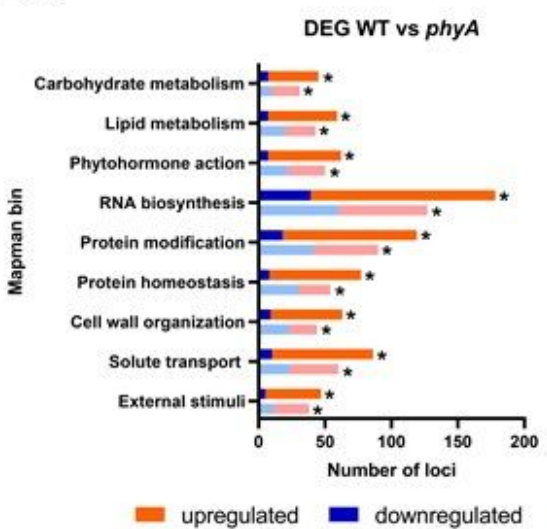

b

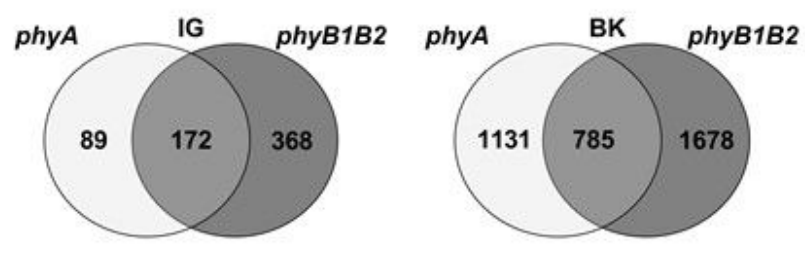

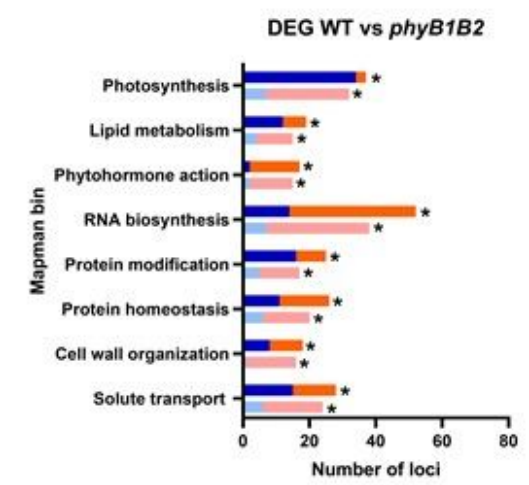

DEG WT vs phyB1B2

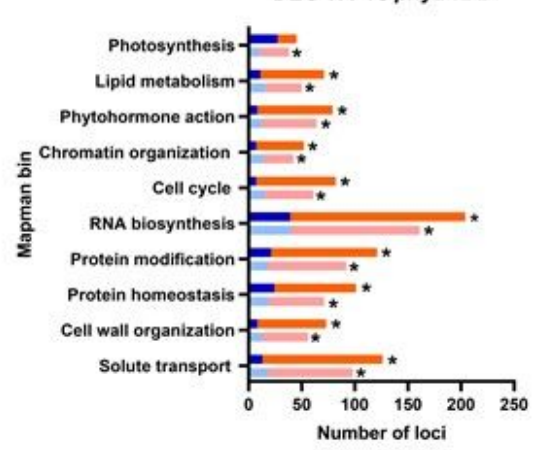

hypermethylated promoters hypomethylated promoters

\section{Figure 1}

PHYA and PHYB1B2 modify the global transcriptomic profile of tomato fruit. (a) Number of differentially expressed genes (DEGs) in phyA and phyB1B2 mutant fruits at immature green (IG) and breaker (BK) stages. (b) Venn diagram showing exclusive and common DEGs in phyA and phyB1B2 mutants in both developmental stages. (c) Functional categorization of all DEGs and those DEGs with differentially methylated promoters (DMPs) in both analysed genotypes and stages. Only categories corresponding to 
at least $2 \%$ of the DEGs or DMPs in each comparison are shown (asterisks). Up- and downregulated genes are indicated in red and blue, respectively. Loci with hyper- and hypomethylated promoters are indicated in light red and light blue, respectively. DEGs and DMPs show statistically significant differences $(F D R<0.05)$ relative to $W T$.

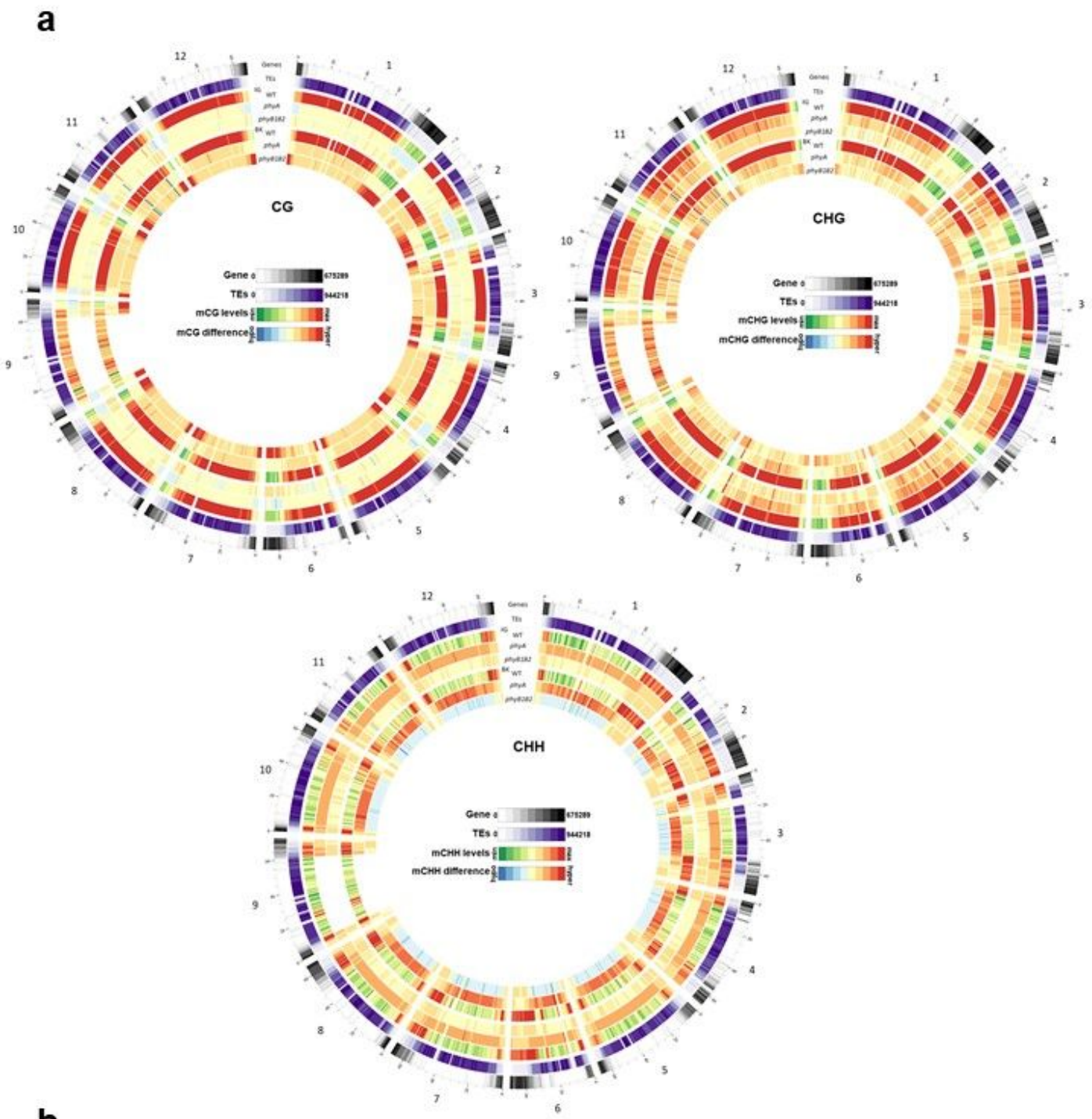

b

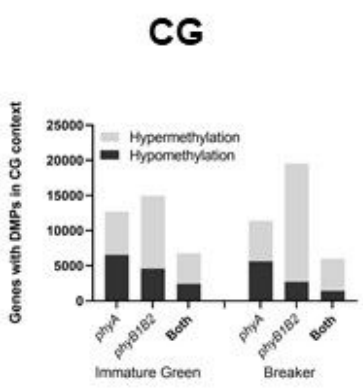

\section{CHG}

$\mathrm{CHH}$
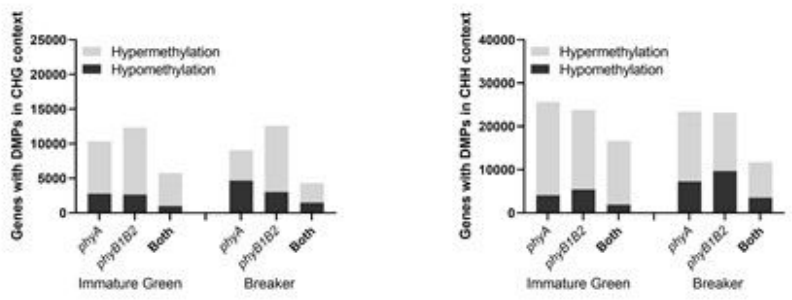

Figure 2 
PHYA and PHYB1B2 regulate the tomato fruit epigenome. (a) Density plot of genes, transposable elements (TEs) and $\mathrm{mC}$ in all contexts ( $\mathrm{mCG}, \mathrm{mCHG}, \mathrm{mCHH}$ ) for the WT genotype. Global methylation changes for phyA and phyB1B2 in comparison with the wild type (WT) at the immature green (IG) and breaker (BK) stages are shown (bin size, $1 \mathrm{Mb}$ ). Gene and TE densities were estimated according to the number of nucleotides covered per million. The methylation levels in the $\mathrm{CG}, \mathrm{CHG}$ and $\mathrm{CHH}$ contexts are $40-90 \%, 25-80 \%$ and $10-30 \%$, respectively. The $\mathrm{mC}$ difference was relative to the corresponding WT fruit stage within a $-5 \%$ (hypomethylated) $\nabla$ range $\nabla+5 \%$ (hypermethylated). (b) Number of genes with differentially methylated promoters (DMPs, 2 kb upstream transcription start site) in phyA, phyB1B2 and both mutants. Hyper- and hypomethylation are indicated by grey and darker-coloured bars, respectively. DMPs show statistically significant differences (FDR < 0.05$)$ relative to WT. 


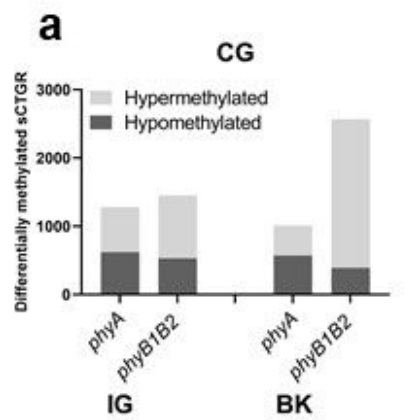

b
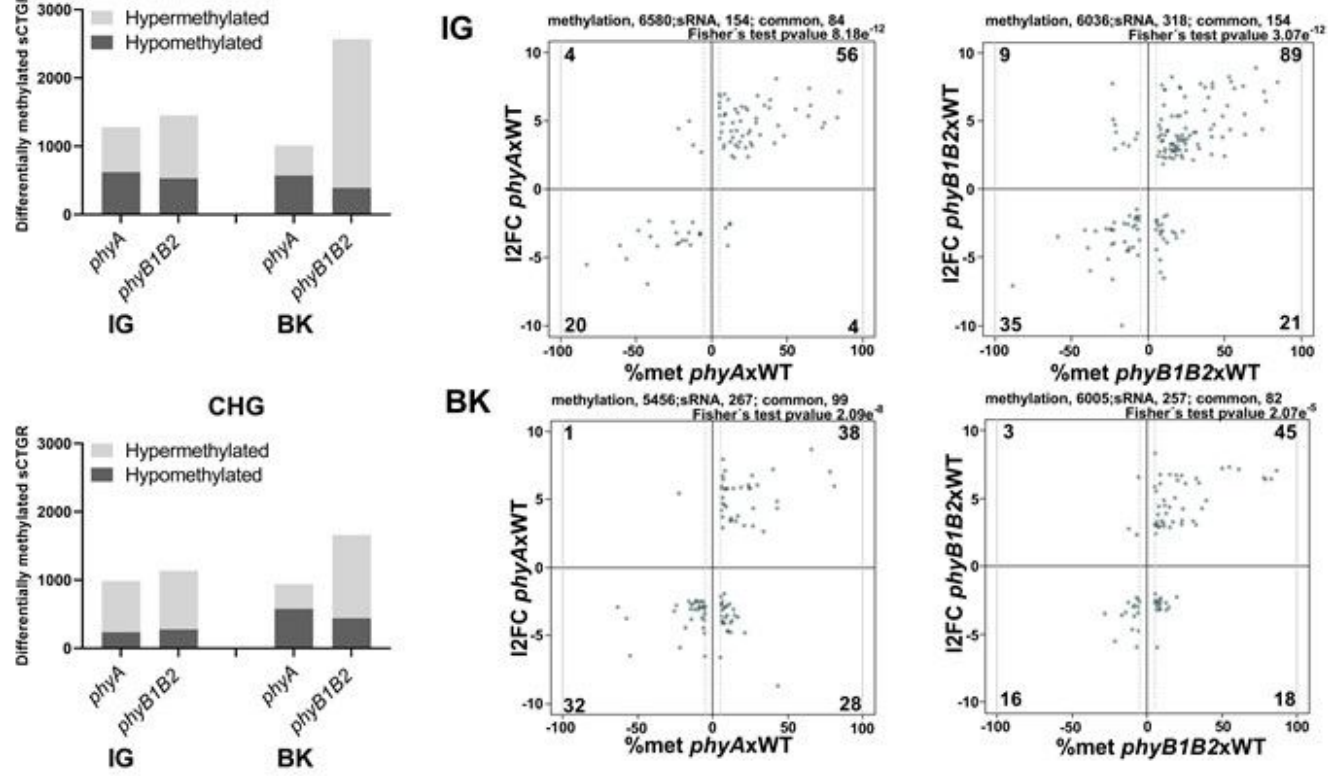

BK
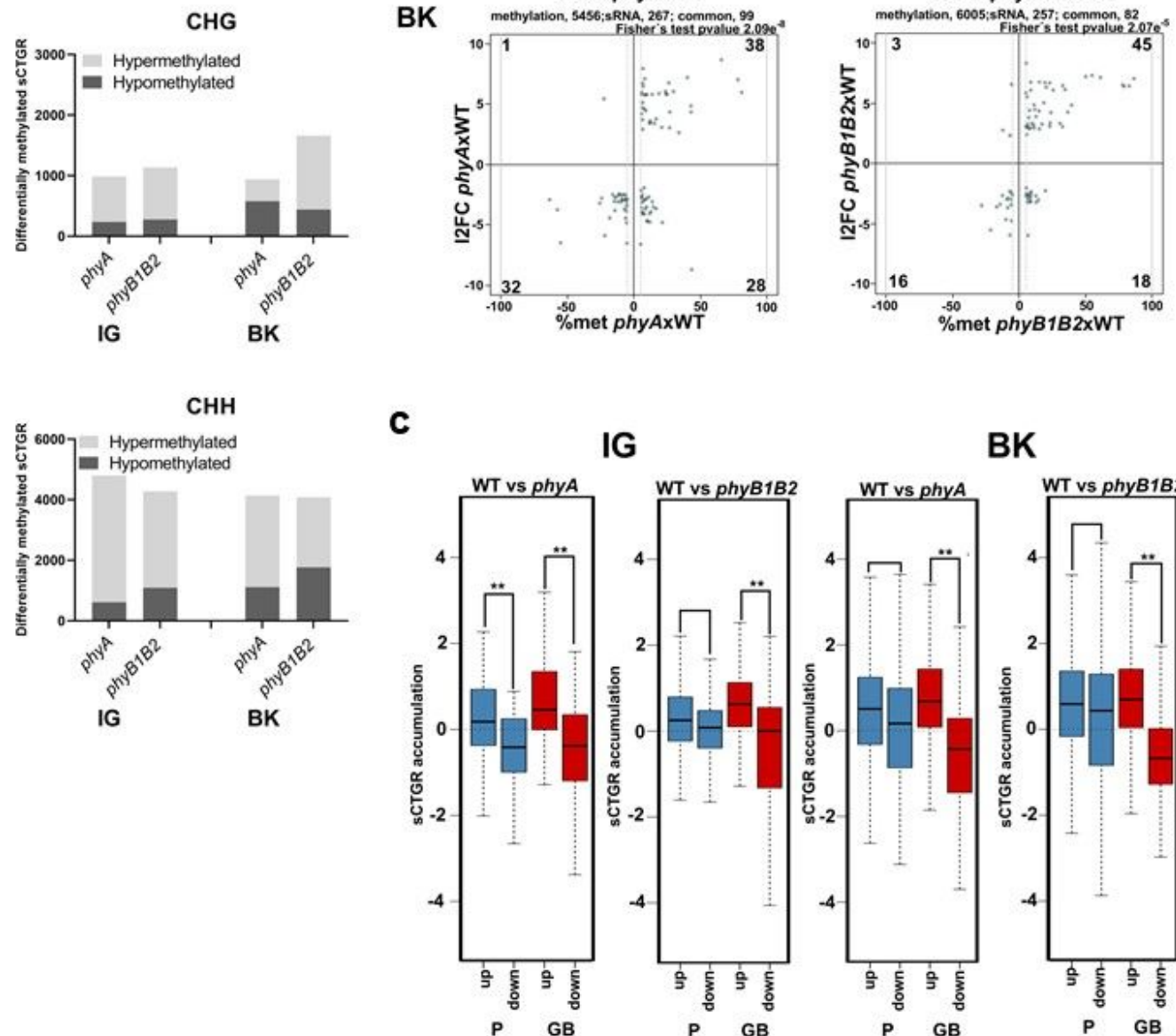

IG

BK
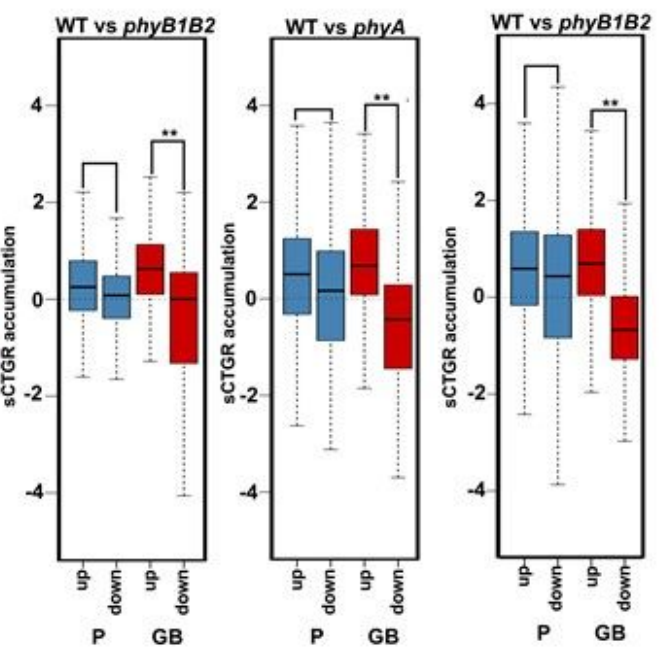

\section{Figure 3}

Phytochrome deficiency impacts the sRNAome profile. (a) Total number of differentially methylated sRNA cluster-targeted genome regions (sCTGRs). (b) Scatter plots show the relationship between the differential accumulation of cluster sRNAs and a minimum of $5 \%$ differential methylation of their sCTGRs. The result of Fischer's test for the association of the two datasets is shown $(p \leq 2.07 e-5)$. (c) Boxplots show changes in the accumulation of cluster sRNAs in promoter (P, 2 Kb upstream of the $5^{\prime}$ UTR 
end) and gene body (GB) regions for up- and downregulated DEGs. Asterisks indicate statistically significant differences by the Wilcoxon-Mann-Whitney test (** $p<0.0001)$. All results represent the comparison of phyA and phyB1B2 to the wild type in immature green (IG) and breaker (BK) fruit stages.
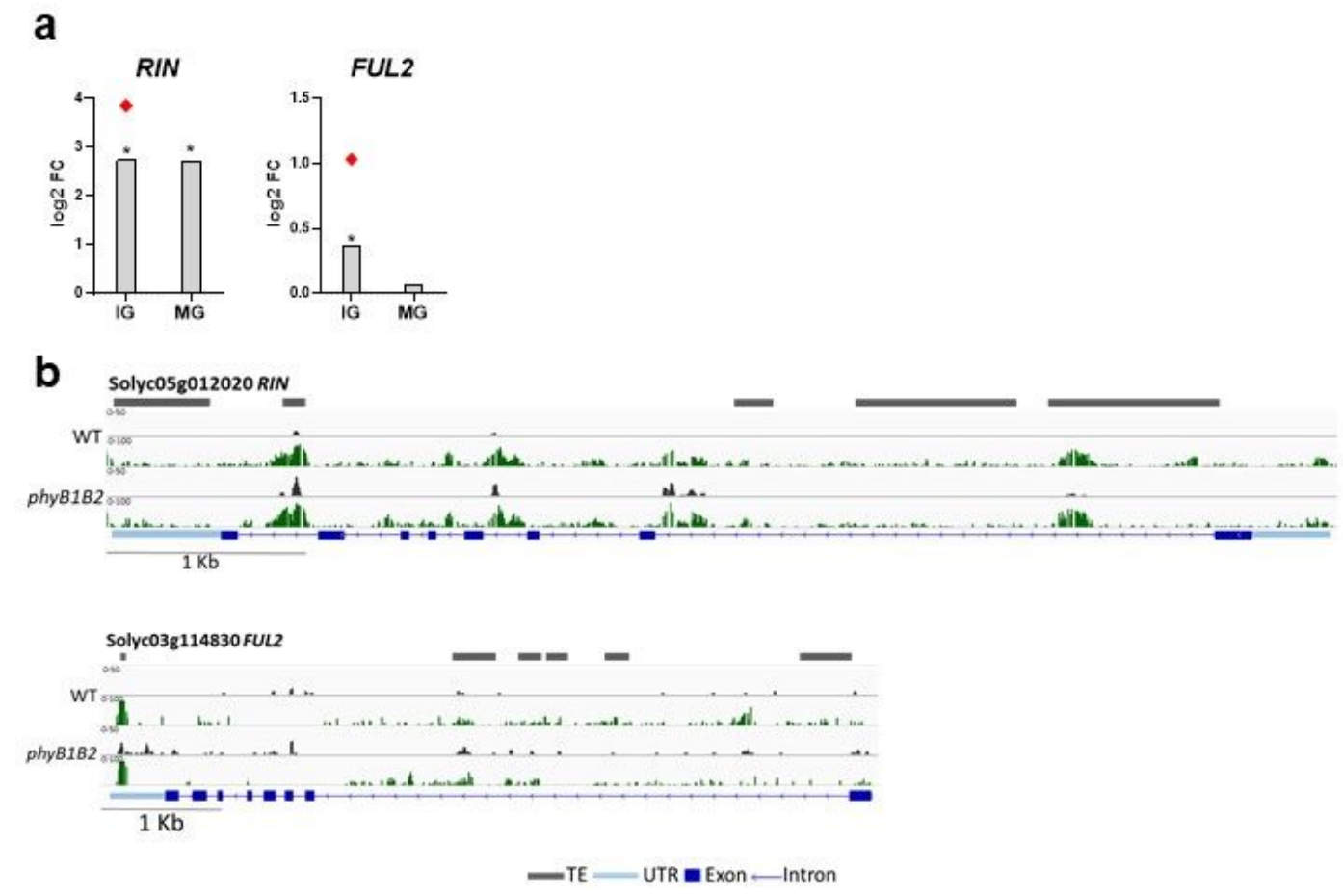

\section{Figure 4}

Methylation across promoter and gene body regions differentially affects gene expression. (a) Relative expression of RIPENING INHIBITOR (RIN) and FRUITFULL 2 (FUL2) in immature green (IG) and mature 
green (MG) fruits from phyB1B2 determined by RT-qPCR. Red dots indicate data from RNA-seq in the same stage. Expression levels represent the mean of at least three biological replicates and are relative to the wild type (WT). Asterisks indicate statistically significant differences by the two-tailed Student's t test compared to WT (* $p<0.05$ ). (b) Differential gene body methylation (green bars) and sRNA accumulation (black bars) within RIN and FUL2 in IG fruits from the phyB1B2 and WT genotypes.

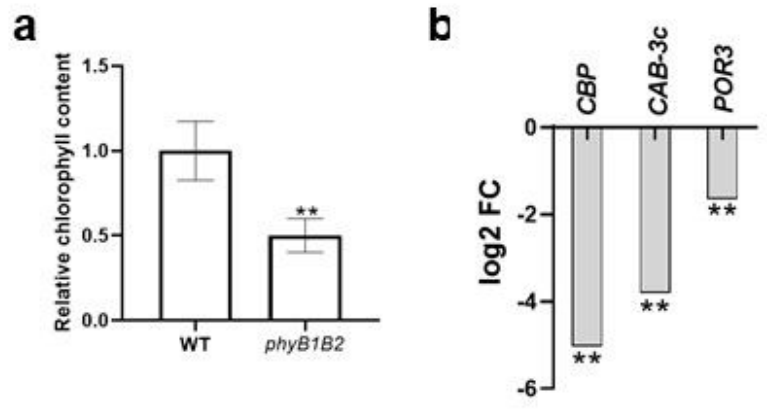

C
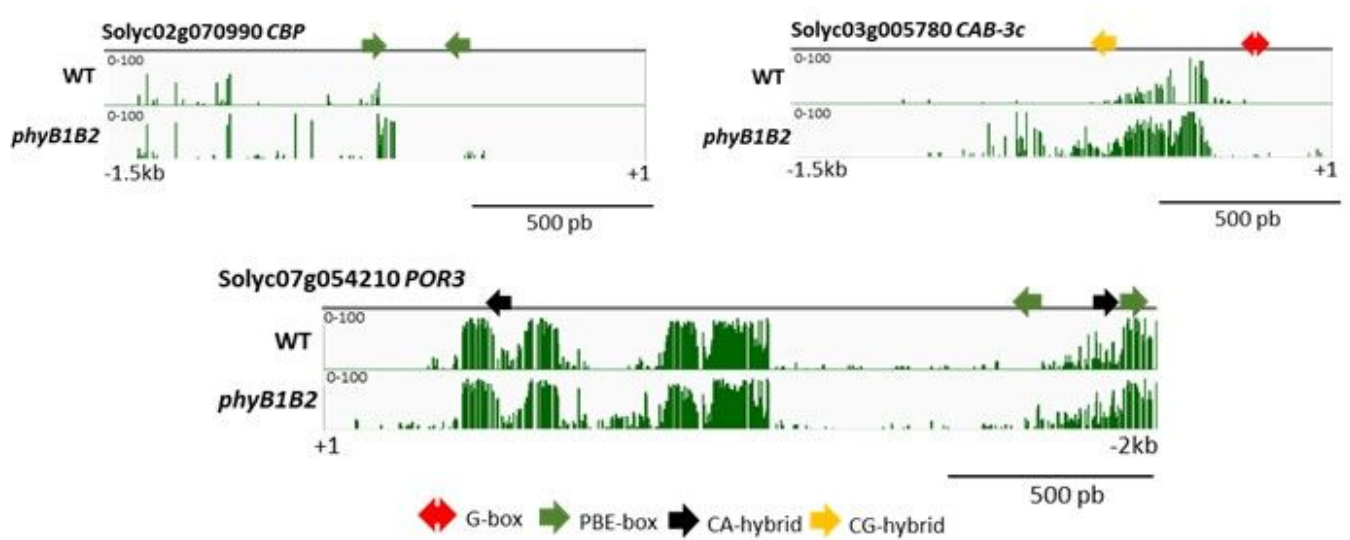

Figure 5 
PHYB1/B2-dependent methylation regulates fruit chlorophyll. (a) Relative content of total chlorophyll in IG fruits from phyB1B2 and WT genotypes. Values represent the mean of at least three biological replicates. Asterisks indicate statistically significant differences by the two-tailed Student's t test between genotypes (** $p<0.01$ ). (b) Relative expression of CHLOROPHYLL A/B BINDING PROTEINs (CBP and CAB3c) and PROTOCHLOROPHYLLIDE OXIDOREDUCTASE 3 (POR3) in IG fruits from phyB1B2 determined by RNA-seq. Expression levels represent the mean of at least three biological replicates and are relative to WT. Asterisks indicate statistically significant differences compared to WT (* FDR $\leq 0.05$ ). (c) Differential promoter methylation in CBP, CAB-3C and POR3 in IG fruits from the phyB1B2 and WT genotypes. HY5 and PIF transcription factor binding motifs are denoted with arrows. 
a
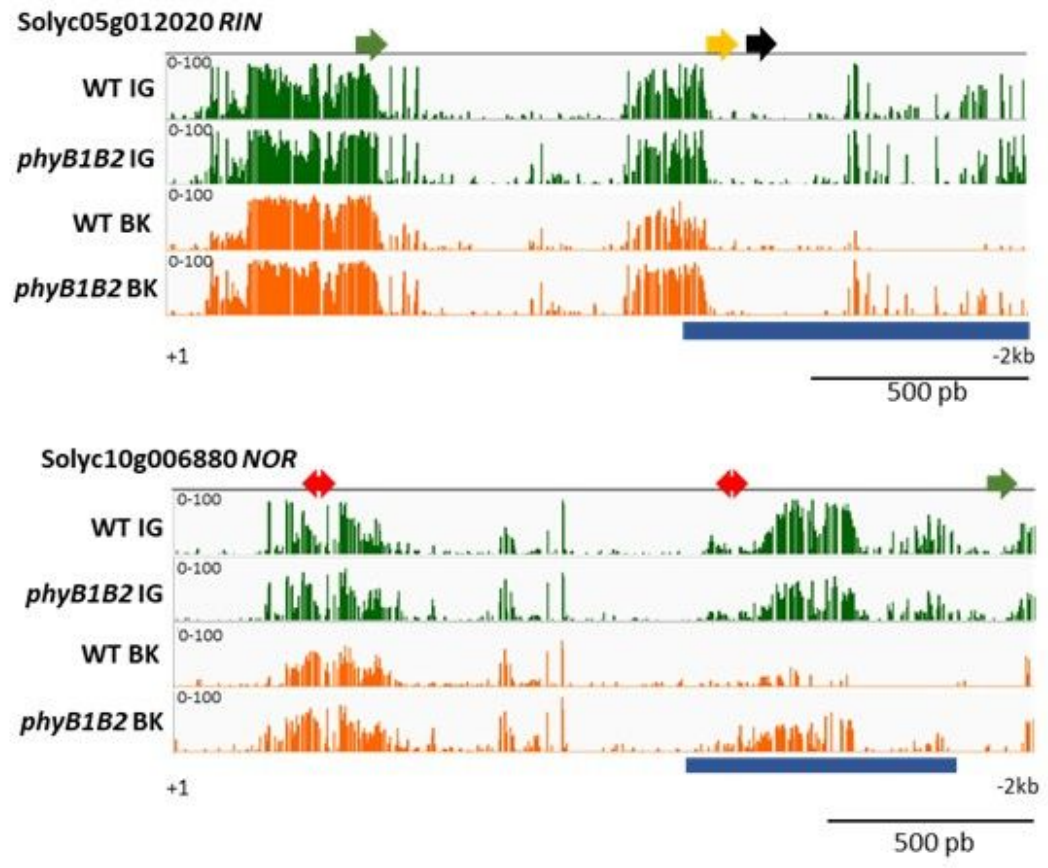

Solyc02g077920 CNR

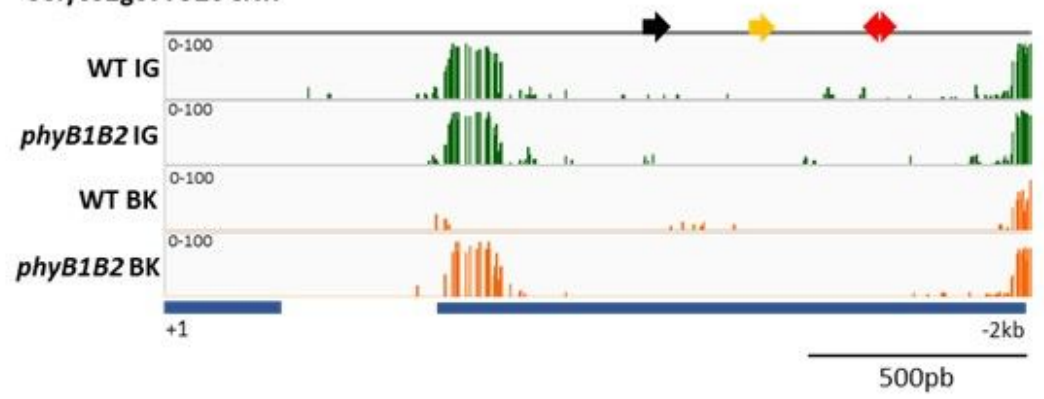

Solyc03g044300 AP2a

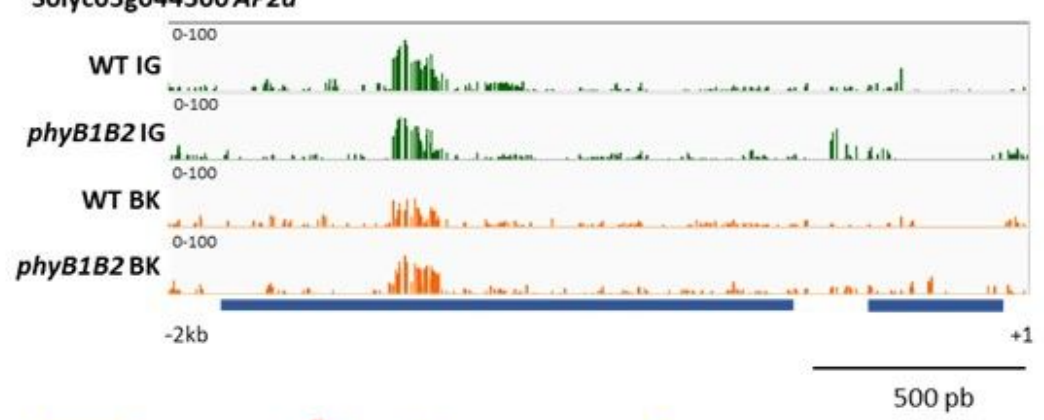

RIN-binding motif $\overrightarrow{\text { G-box }} \Rightarrow$ PBE-box ca-hybrid $\Rightarrow$ CG-hybrid b
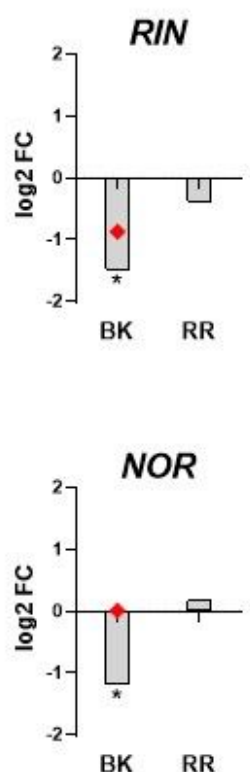

BK RR
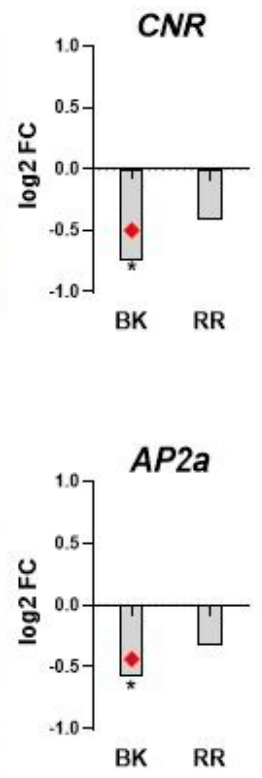

\section{Figure 6}

PHYB1/B2-dependent methylation regulates master ripening-associated transcription factors. (a) Differentially methylated promoters of the RIPENING INHIBITOR (RIN), NON-RIPENING (NOR), COLORLESS NORIPENING (CNR) and APETALA 2a (AP2a) loci between the phyB1B2 and wild-type (WT) genotypes. Green and orange indicate total $\mathrm{mC}$ in immature green (IG) and breaker (BK) fruits, respectively. HY5 and PIF transcription factor binding motifs are denoted with arrows. Thick blue lines 
indicate RIN binding sites according to ChIP-seq data27. (b) Relative expression from the RT-qPCR assay of genes encoding master ripening transcription factors in BK and red ripe (RR) fruits from phyB1B2. Red dots indicate data from RNA-seq in the same stage. Expression levels represent the mean of at least three biological replicates and are relative to WT. Asterisks indicate statistically significant differences by twotailed Student's t test compared to WT (* $p<0.05)$.

a

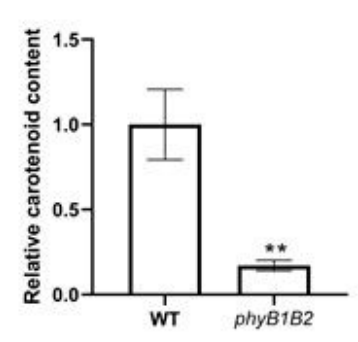

C
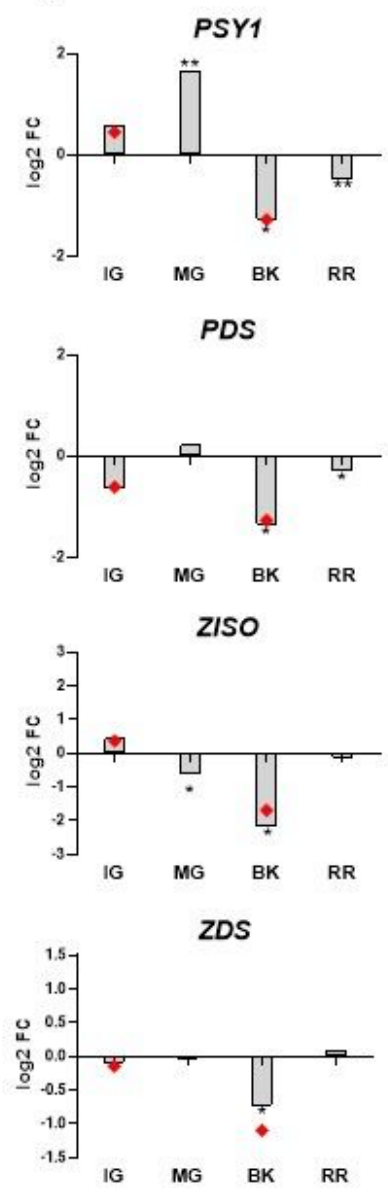

b

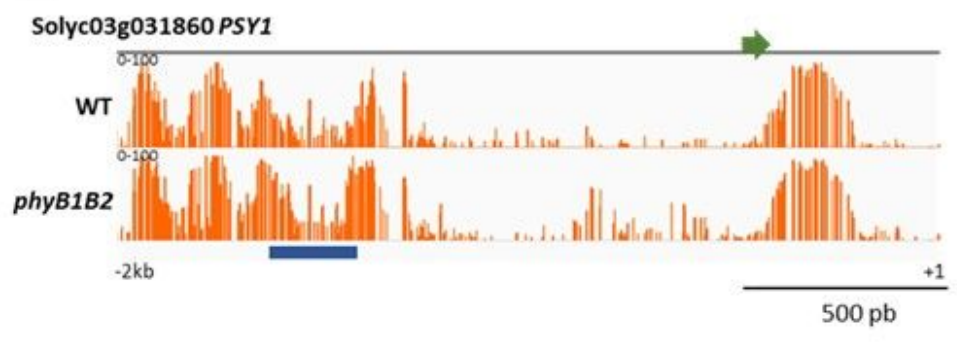

Solyc03g123760 PDS
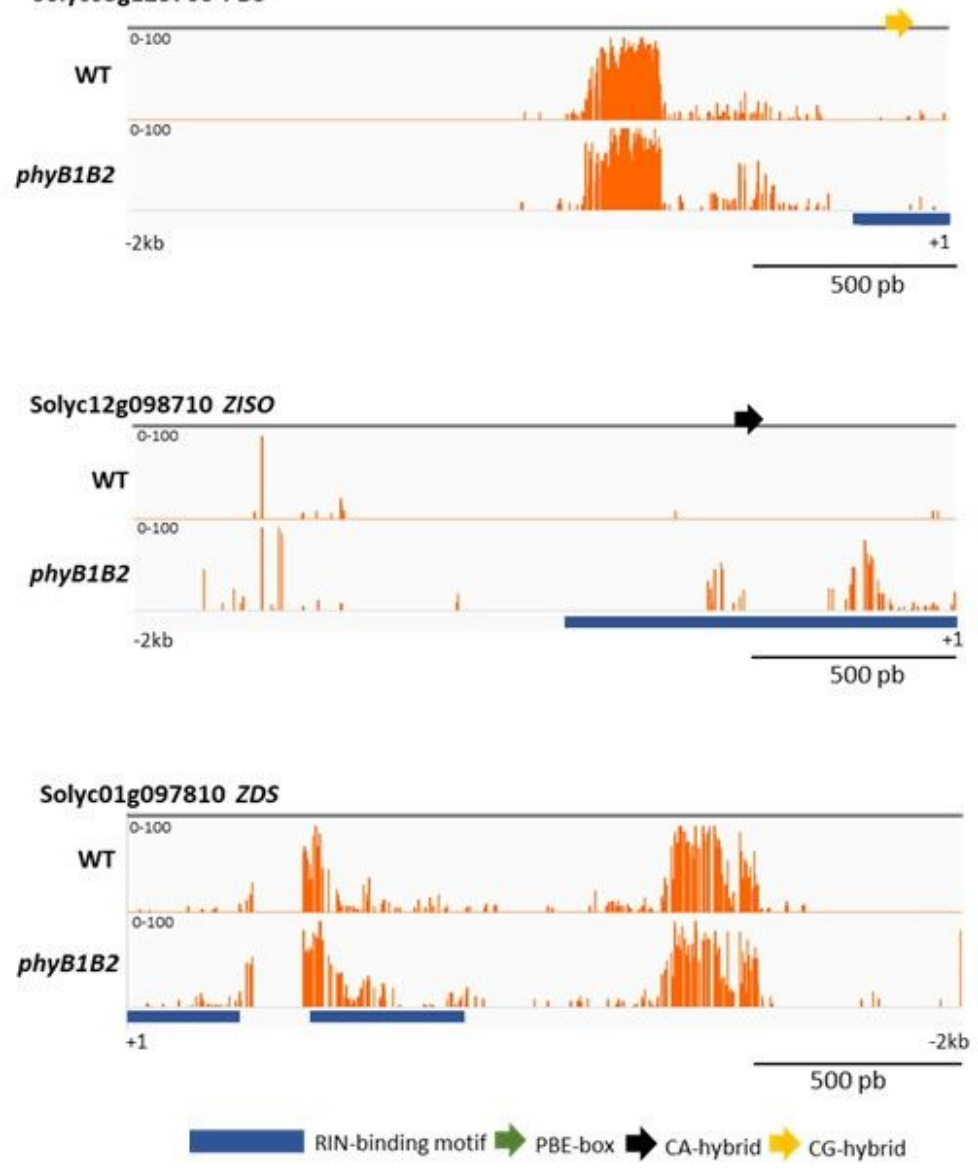

Figure 7 
PHYB1/B2-dependent methylation regulates master ripening-associated transcription factors. (a) Differentially methylated promoters of the RIPENING INHIBITOR (RIN), NON-RIPENING (NOR), COLORLESS NORIPENING (CNR) and APETALA 2a (AP2a) loci between the phyB1B2 and wild-type (WT) genotypes. Green and orange indicate total $\mathrm{mC}$ in immature green (IG) and breaker (BK) fruits, respectively. HY5 and PIF transcription factor binding motifs are denoted with arrows. Thick blue lines indicate RIN binding sites according to ChIP-seq data27. (b) Relative expression from the RT-qPCR assay of genes encoding master ripening transcription factors in BK and red ripe (RR) fruits from phyB1B2. Red dots indicate data from RNA-seq in the same stage. Expression levels represent the mean of at least three biological replicates and are relative to WT. Asterisks indicate statistically significant differences by twotailed Student's t test compared to WT ( $\left.{ }^{*} p<0.05\right)$. 
a

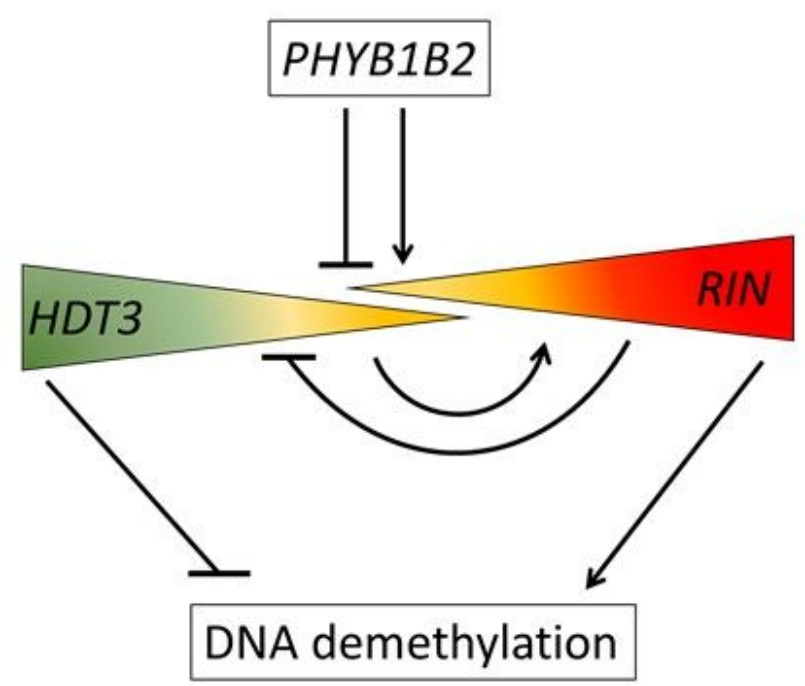

b

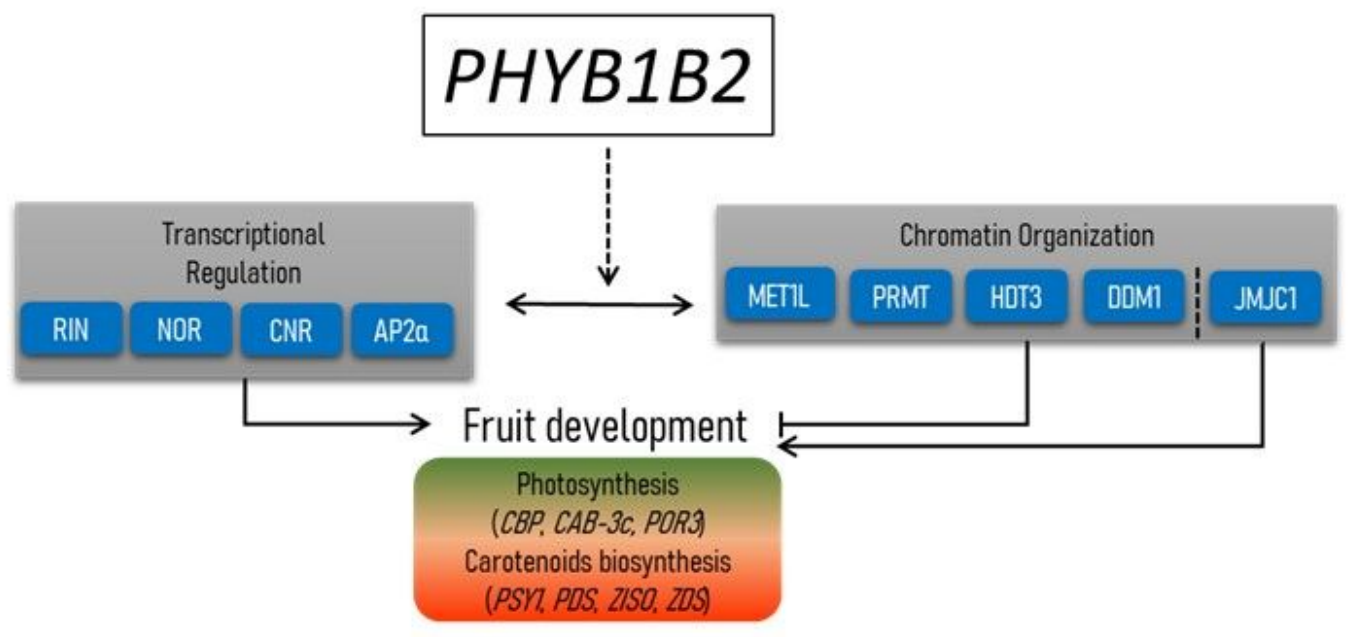

Figure 8

Model of epigenetic mechanisms involved in fruit development regulation triggered by PHYB1B2. (a) PHYB1B2 contributes to the reciprocal regulation between HDT3 and RIN through the inhibition and induction of HDT3 and RIN expression, respectively. Increases in RIN expression during fruit maturation inhibit HDT3 levels triggering DNA demethylation and consequently fruit ripening. (b) Active PHYB1B2 regulates the expression of genes associated with DNA methylation (MET1L), chromatin remodelling 
(DDM1) and histone modifiers (PRMT and HDT3), promoting overall fruit DNA demethylation during the ripening process. PHYB1B2-mediated methylation reprogramming results in the upregulation of master TFs, which in turn trigger ripening and carotenogenesis.

\section{Supplementary Files}

This is a list of supplementary files associated with this preprint. Click to download.

- SupplementaryTable1.xIsx

- SupplementaryTable2.xlsx

- SupplementaryTable3.xlsx

- SupplementaryTable4.xlsx

- SupplementaryTable5.xlsx

- SupplementaryTable6.xIsx

- SupplementaryTable7.xlsx

- SupplementaryTable8.xlsx

- SupplementaryTable9.xlsx

- SupplementaryTable10.xIsx

- SupplementaryTable11.xlsx

- SupplementaryTable12.xIsx

- SupplementaryTable13.xlsx

- SupplementaryTable14.xIsx

- SupplementaryTable15.xlsx

- SupplementaryTable16.xlsx

- SupplementaryTable17.xIsx

- SupplementaryTable18.xlsx

- SupplementaryTable19.xlsx

- SupplementaryTable20.xlsx

- SupplementaryTable21.xlsx

- SupplementaryFigure1final.jpg

- SupplementaryFigure21.jpg

- SupplementaryFigure3.jpg

- SupplementaryFigure4.jpg

- SupplementaryFigure5.jpg

- SupplementaryFigure6.jpg 